\title{
The component parts of bacteriophage virions accurately defined by a machine-learning approach built on evolutionary features.
}

Tze Y. Thung ${ }^{1,2,+}$, Murray E. White ${ }^{1,2,+}$, Wei Dai ${ }^{1,3,+}$, Jonathan J. Wilksch ${ }^{1,2}$, Rebecca S. Bamert $^{1,2}$, Andrea Rocker ${ }^{1}$, Christopher J Stubenrauch ${ }^{1,2}$, Daniel Williams ${ }^{1,2}$, Cheng Huang ${ }^{4}$, Ralf Schittelhelm ${ }^{4}$, Jeremy J. Barr ${ }^{2,5}$, Eleanor Jameson ${ }^{6}$, Sheena McGowan ${ }^{1,2}$, Yanju Zhang ${ }^{3}$, Jiawei Wang ${ }^{1,2, *}$, Rhys A. Dunstan ${ }^{1,2, *} \&$ Trevor Lithgow ${ }^{1,2, *}$

1. Infection \& Immunity Program, Biomedicine Discovery Institute and Department of Microbiology, Monash University, Clayton, Australia.

2. Centre to Impact AMR, Monash University, Clayton, Australia.

3. School of Computer Science and Information Security, Guilin University of Electronic Technology, Guilin 541004, China

4. Monash Proteomics \& Metabolomics Facility, Biomedicine Discovery Institute and Department of Biochemistry and Molecular Biology, Monash University, Clayton, Australia.

5. School of Biological Sciences, Monash University, Clayton, Australia.

6. School of Life Sciences, University of Warwick, Gibbet Hill Road, Coventry CV4 7AL, UK

+ - These authors contributed equally.

* - Correspondence should be addressed to: trevor.lithgow@ monash.edu, rhys.dunstan@monash.edu, jiawei.wang@monash.edu

Keywords: antimicrobial resistance, phage therapy, bacteriophage, artificial intelligence 


\section{ABSTRACT}

3 Antimicrobial resistance (AMR) continues to evolve as a major threat to human health and new

4 strategies are required for the treatment of AMR infections. Bacteriophages (phages) that kill

5 bacterial pathogens are being identified for use in phage therapies, with the intention to apply

6 these bactericidal viruses directly into the infection sites in bespoke phage cocktails. Despite the

7 great unsampled phage diversity for this purpose, an issue hampering the roll out of phage

8 therapy is the poor quality annotation of many of the phage genomes, particularly for those from

9 infrequently sampled environmental sources. We developed a computational tool called STEP ${ }^{3}$

10 to use the "evolutionary features" that can be recognized in genome sequences of diverse phages.

11 These features, when integrated into an ensemble framework, achieved a stable and robust

12 prediction performance when benchmarked against other prediction tools using phages from

13 diverse sources. Validation of the prediction accuracy of STEP $^{3}$ was conducted with high-

14 resolution mass spectrometry analysis of two novel phages, isolated from a watercourse in the

15 Southern Hemisphere. STEP $^{3}$ provides a robust computational approach to distinguish specific

16 and universal features in phages to improve the quality of phage cocktails, and is available for

17 use at http://step3.erc.monash.edu/.

\section{IMPORTANCE}

20 In response to the global problem of antimicrobial resistance there are moves to use

21 bacteriophages (phages) as therapeutic agents. Selecting which phages will be effective

22 therapeutics relies on interpreting features contributing to shelf-life and applicability to

23 diagnosed infections. However, the protein components of the phage virions that dictate these

24 properties vary so much in sequence that best estimates suggest failure to recognize up to $90 \%$ of

25 them. We have utilised this diversity in evolutionary features as an advantage, to apply machine

26 learning for prediction accuracy for diverse components in phage virions. We benchmark this

27 new tool showing the accurate recognition and evaluation of phage components parts using

28 genome sequence data of phages from under-sampled environments, where the richest diversity

29 of phage still lies. 


\section{INTRODUCTION}

3 Antimicrobial resistance (AMR) has risen to prominence as a major threat to human health (1,

4 2) and new strategies are required for the treatment of AMR infections (3-5). For example, the

5 Centers for Disease Control and Prevention have identified several species of microbes as

6 "Urgent" threats to human health by virtue of their AMR phenotypes, including Escherichia

$7 \quad$ coli and Enterococcus faecalis. As another prime example of one of these, the carbapenem-

8 resistant Enterobacteriaceae (CRE), Klebsiella pneumoniae infections represent a key target

9 for new therapeutics to treat AMR infections (3-5). Bacteriophages (phages) that kill bacterial

10 pathogens such as Klebsiella are being identified for use in phage therapies, with the intention

11 to apply these bactericidal viruses directly into the infection sites. Careful consideration is

12 needed in selecting the phages for use in therapeutic cocktails (4-6), considerations made

13 difficult because annotation of phage genomes is poor $(7,8)$, potentially obscuring phages with

14 therapeutic potential. For example, while structural motifs are now known (9) that will promote

15 phage virion stability (i.e. shelf-life), only with correct annotation of the major capsid, minor

16 capsid and other proteins involved can structural motifs be identified and evaluated.

18 Phage therapy has re-emerged because of its potential treatment for antimicrobial-resistant

19 infections, and a common protocol for treatments is to select two or more phages for

20 combination into a treatment cocktail (4-6). An ongoing issue is the establishment of criteria

21 used for selection of appropriate phages for a cocktail, to enhance production and maximize

22 efficacy, and to circumvent issues of phage-resistance and collateral induction of further drug-

23 resistance in the infection sites $(4,6)$. The phages used for phage therapy are Caudovirales

24 conforming to a blue-print of an icosahedral protein capsid housing the phage genome, and a

25 tail composed of 20-40 protein components (10). The tail of these phages can be considered as

26 a complex piece of molecular machinery, with component parts of the tail recognizing and

27 docking to a species-specific receptor on the host bacterium $(11,12)$. Penetration of the host

28 cell envelope depends on other components of the tail, which can have enzymatic functions to

29 locally hydrolyze each of the distinct layers of the bacterial envelope (12-14). An ultimate goal

30 for the development of personalized phage therapy is the recognition of all of these components

31 from genome sequence data, so that bespoke phage could be selected for specific therapeutic

32 purposes $(5,6)$. However, the annotation of phage genomes is poor, potentially obscuring

33 important features contributed by some component parts such as contributions to virion

34 stability and shelf-life, host-range and bacterial cell lysis $(7,8,15)$. 


\section{RESULTS AND DISCUSSION}

3 Currently phage genomes are assessed by tools such as multiPhATE (15) which provides a

4 bioinformatics pipeline for functional annotation using sequence-based queries. The annotation

5 accuracy of multiPhATE is limited by the extreme sequence diversity in phage genomes, likely

6 due to the rapid evolutionary rates of phages (16). This limitation has been addressed to some

7 extent with a neural network-based predictor iVIREONS (17) and further tools such as PVPred

8 (18), PVP-SVM (19), PhagePred (20), Pred-BVP-Unb (21) and PVPred-SCM (22). However,

9 recent evaluation of these tools in phage protein prediction showed less than satisfactory

10 performance (23). We developed an ensemble predictor, $\mathrm{STEP}^{3}$, to accurately call the protein

11 components of phage virions and visualize their predicted function-based relationships (Fig. 1).

13 STEP $^{3}$ extracted information from Position-Specific Scoring Matrix (PSSM) data (Fig. 1a), an 14 approach that tracks protein evolutionary histories $(24,25)$. In machine-learning evaluation of

15 protein sequences, "evolutionary features" refer to information within the amino acid sequences

16 that conceptually traces the evolutionary history of proteins, and their use often identifies highly

17 informative patterns $(24,25)$. Indirectly, these evolutionary features effectively capture structural

18 as well as physicochemical properties. STEP $^{3}$ includes data visualization capabilities to

19 document relationships between virion components where the sequence similarity is sufficiently

20 strong to identify high confidence homologs from other phages (Fig. 1b, Supplementary Fig. 1).

22 There is power in integrating individual models within an ensemble framework for more robust

23 and stable predictions: trained with an individual model alone (AAC-PSSM), predictions

24 perform well with the 5-fold cross-validation test (Supplementary Table 1), but ranked only fourth using the independent test (Supplementary Table 2). In contrast, combined with other models into the ensemble model of $\mathrm{STEP}^{3}$, to draw on the best elements from all of the individual models (Fig. 1a), the best prediction performance ranking was achieved (Supplementary Tables 1,2). In benchmarking against other available predictors, the ensemble $\mathrm{STEP}^{3}$ achieved an improved performance, with the highest sensitivity ( $\mathrm{SN}=0.896$ ), accuracy $(\mathrm{ACC}=0.891)$, F-value $(0.891)$ and Matthews Correlation Coefficient $(\mathrm{MCC}=0.781)$ using the independent test (Supplementary Table 3). The superior performance of $\mathrm{STEP}^{3}$ can be attributed to the integration of more informative evolutionary features, as well as the comprehensive and up-to-date training dataset using experimentally verified inputs. It is worth noting that the BLAST-based predictor, which represents the mode used for genome annotation had the lowest accuracy (ACC) and F-value. This prediction bias is reflected by the extremely unbalanced sensitivity (the lowest) and specificity (the highest) scores, so that the BLAST-based predictor 
1 tended to predict positive samples as being negative. This quantifies and evidences past

observations that pairwise sequence matching methods struggle to predict phage proteins (25).

4 As initial case studies we drew on three accounts published after $\mathrm{STEP}^{3}$ was trained, where

5 phages had been discovered, the genome sequence data deposited for public access, and the

6 protein composition virions had been determined by mass spectrometry. The mass spectrometry

7 data is crucial as it enables a discrimination between false positive (FP; predicted but not present

8 by mass spectrometry of the virion) and true positive (TP; predicted and found present by mass

9 spectrometry of the virion). Phage vB_EfaS_271 infects Enterococcus faecalis (26), phage

10 vB_PatM_CB7 infects Pectobacterium atrosepticum (27), and phage vB_Eco4M-7 infects

11 enteropathogenic Escherichia coli (28). STEP $^{3}$ was benchmarked against equivalent predictors:

12 PVPred, PVP-SVM, PVPred-SCM and Pred-BVP-Unb (Fig. 2). STEP 3 provided the greatest set

13 of true positive predictions for each of the three phages, predicting 9 of the 12 virion components

14 for phage vB_EfaS_271, 23 of the 26 protein components for phage vB_PatM_CB7 and 24 out

15 of 33 components of the phage vB_Eco4M-7 virions. Making low FP predictions on each phage,

$16 \mathrm{STEP}^{3}$ maintained a good balance between TP and FP and showcased robust prediction

17 performance across the test cases. In the case of phage vB_PatM_CB7, where mass spectrometry

18 data had shown the number of non-virion proteins is more than eight times as many as that of

19 virion proteins, $\mathrm{STEP}^{3}$ generated an equal number of FP as that of TP. In this extreme case,

$20 \mathrm{STEP}^{3}$ correctly predicts 23 out of 26 virion proteins with a false positive rate of $10.1 \%$

$21(23 / 227)$.

23 Oftentimes candidate phages that kill pathogens are isolated from hospital waste-water sources

24 for their use in phage therapy $(29,30)$. This raises the issue of potential over-sampling of a common environmental source (i.e. wastewater) for phages, potentially limiting discovery of other, valuable phages and also potentially biasing the capability of predictors like STEP ${ }^{3}$.

27 Therefore, as a further proof of principle test for $\mathrm{STEP}^{3}$ we sampled a natural watercourse with a 28 strain of drug-resistant and hypervirulent Klebsiella pneumoniae as host. The Merri Creek, 29 which forms a part of the larger Merri catchment, lies within Wurundjeri Woi Wurrung people's 30 traditional homelands. Phages isolated from two separate sampling sites were characterized 31 initially by genome sequencing and named in Woi wurrung language Merri-merri-uth nyilam 32 marra-natj (MMNM) and Merri-merri baany-a bundha-natj (MMBB). These names translate as

33 "Dangerous Merri lurker" and "Merri water biter", respectively, in English.

35 Comparative genomic analysis revealed Klebsiella phages MMNM (Supplementary Fig. 2) and 36 MMBB (Supplementary Fig. 3) to be distinct from previously sampled phages. In the case of 
1 MMNM, some similarities can be seen to phages belonging to the Jedunavirus genus according

2 to the most recent International Committee on Taxonomy of Viruses (ICTV) classification, but

3 the branch lengths on the tree designate diversity within this small group, comprising only eight

4 phages in the NCBI database (Fig 3a). Relatives of MMNM, isolated from hospital wastewater

5 in Russia, showed considerable diversity in gene content and arrangement (Fig 3b). Most

6 notably, MMNM encodes several genes that are absent in many of the other sequenced

7 Jedunaviruses, including previously uncharacterised proteins MMNM_5, MMNM_6

8 MMNM_45, MMNM_51, MMNM_56, MMNM_57 and the putative polynucleotide kinase

9 protein MMNM_50. Conversely, MMNM lacks the putative NHN endonuclease-like protein

10 encoded by both vB_KpnM_FZ14 and vB_KpnM_KpV52. Sequence annotations

11 (Supplementary Table 4) suggest that MMNM has a tail structure characteristic of Myoviridae,

12 including a baseplate protein (MMNM_21), a baseplate J-like protein (MMNM_23) and the

13 base-plate wedge protein (MMNM_26). In high resolution structural analyses of the Myoviridae

14 phage T4, each virion has 6 molecules of each of these proteins and 1-3 molecules per virion of

15 the hub proteins to which the baseplate is attached $(31,32)$.

MMBB belongs to the Webervirus genus, a group of phages that exclusively target Klebsiella species (Supplementary Fig 3). MMBB is distinct from the other phages in this genus, with its closest relationship being to a phage isolated in China called vB_KpnS_GH-K3 (also called phage GH-K3) (33). Highlighting their differences, MMBB and GH-K3 show regions of diversity in gene content and arrangement, this is observed for the gene encoding MMBB_16, a putative AP2/HNH endonuclease previoulsy found only in a small number of other Siphoviridae phages including the Escherichia phage vB_EcoS_ESCO41 and Escherichia phage CJ19 (Supplementary Fig 2). Additional differences are seen in a contiguous cluster of four genes encoding hypothetical proteins (MMBB_45-MMBB_48) that are absent in GH_K3.

Phenotypic characterization of the phages on lawns of $K$. pneumoniae (Methods) showed that the plaque size for MMNM was smaller than MMBB (Fig 4a), and with liquid cultures of $K$. pneumoniae (Methods) that MMNM had a shorter latent period (L) before host cell death as determined by one-step growth curves (Fig 4b). Electron microscopy revealed that MMNM has an icosahedral head and a tail tube of $\sim 54 \mathrm{~nm}$ capped with a $\sim 30 \mathrm{~nm}$ baseplate to generate thick and straight tails (Fig 4c). The baseplate structure evident in MMNM (Fig 4c) is similar to that seen for the T4 phage (31), which serves as a paradigm for the Myoviridae (34) (Fig 4d). By contrast, MMBB has $\sim 200 \mathrm{~nm}$ long, slender and flexible tails (Fig 4c). The flexible, noncontractile tail tube designate MMBB as a phage of Siphoviridae-like viruses (Fig 4d), consistent with genome annotation data (Supplementary Table 5). 
2 To directly test $\mathrm{STEP}^{3}$ prediction capability on the novel phages MMNM and MMBB, the

3 protein components contributing structurally to the virions were determined by high-

4 performance mass spectrometry $(35,36)$. To this end, samples of each virion were purified using

5 caesium chloride gradients. The MMNM virion is composed of 25 protein components

6 (Supplementary Table 6). Assuming a similar stoichiometry between MMNM virions and the

7 paradigm for Myoviridae, phage T4 virions, the identification of the lytic transglycosylase

8 MMNM_19 suggests that the proteomic analysis is sensitive enough to detect 3 or fewer

9 molecules per virion (31). From evaluation of the predicted proteins within the phage genomes,

10 together with this mass spectrometry data, the MMNM genome encodes 25 structural proteins

11 that serve as components of the virion and 42 proteins that would be expressed after infection of

12 the host, to drive phage replication (Fig 5a).

14 STEP $^{3}$ successfully predicted 22 out of the 25 MMNM virion proteins (Fig 5b, Supplementary

15 Table 7). The other predictors gave poorer outcomes with these diverse protein sequences. For

16 example, second to $\mathrm{STEP}^{3}$ was iVIREONS which identified 19 virion proteins, but iVIREONS

17 also generated the largest number of false positives, 14, consistent with its high false positive

18 prediction rate in the independent tests (Supplementary Table 3). In one case, the initial STEP ${ }^{3}$

19 analysis made a false-negative prediction that was highly informative. The phage polynucleotide

20 kinase (PNK) is an enzyme that has been previously assumed to be a non-virion protein, and the

21 sequence was therefore included in that (non-virion) dataset from which STEP$^{3}$ was trained.

22 However, mass spectrometry identified the putative PNK protein MMNM_50 as a component of

23 the virion Supplementary Table 6). Note, an equivalent result was achieved with the prediction

24 for MMBB: protein MMBB_64 was detected by mass spectrometry (Supplementary Table 9)

25 and selected by $\operatorname{STEP}^{3}$ (Supplementary Table 8). We suggest that for some phages the PNK

26 remains associated with the packaged genome and is thereby incorporated within the capsid.

27 This suggestion explains the proteomics data herein, reconciles the false-negative prediction by

$28 \mathrm{STEP}^{3}$, and is consistent with the recent observation that the "gp44 ejection protein" is a virion-

29 protein in a Staphylococcus phage $80 \alpha$ bound to genome ends and functioning as a putative PNK

30 would to protect the DNA from degradation upon phage entry into its host (37).

32 High-resolution mass spectrometry of the MMBB virions showed them to be composed of 29

33 protein components (Supplementary Table 9). Thus, the MMBB genome encodes 29 proteins

34 contributing structurally to the virions, and 50 non-virion proteins expressed only after infection

35 in the host bacterium (Fig 4c). For MMBB, STEP ${ }^{3}$ and iVIREONS retrieved 20 and 18 virion 
1 proteins, respectively (Fig 4d, Supplementary Table 8). The other predictors achieved

2 unsatisfactory prediction results, retrieving less than half of the 29 virion proteins.

4 The evolutionary features drawn on by STEP $^{3}$ and iVIREONS are structure-informed, in that the

5 patterns that they recognize are reflections of secondary and tertiary structure, and these patterns

6 can also be used to suggest protein function. For example, a characteristic of the Webervirus has

7 been suggested to be the presence of tail-spike proteins with polysaccharide degrading activity

8 (38), and the sequence of MMBB_78 is suggestive of such a protein, as summarized in

9 Supplementary Fig. 3. Conversely, pairwise sequence assessment is a poor means for recognition

10 and characterization of virion proteins. For both MMNM and MMBB, sequence conservation

11 alone proved the least satisfactory method for predicting phage virion proteins: the BLAST-

12 based predictor recognized only 3 and 6 virion proteins, respectively (Fig 5b, 5d, Supplementary

13 Tables 7, 8). This confirmed the independent test results that the BLAST-based methods

14 commonly used for annotations are a poor means of recognizing and classifying sequence-

15 diverse phage proteins.

17 Some estimates put the number of phage virions in the world at $10^{31}$, suggesting that there is a

18 huge pool of phages that we know little about (39). This encourages a move towards informed

19 bioprospecting for potentially useful phages from under-sampled environments. The effective

20 use of these for therapy and other applications depends on a number of factors, not least of which

21 is the sequence-based choices that must be made to identify novel phages warranting further

22 characterization and potential development into phage therapy. We suggest that application of

$23 \mathrm{STEP}^{3}$ will assist in distinguishing the specific and universal features in phages isolated from

24 under-represented (under-sampled) geographical locations, with impact on the quality of future

25 phage cocktails. Particularly in phage that might be highly divergent in their sequence

26 characteristics, such as the MMNM and MMBB case studies here, $\mathrm{STEP}^{3}$ can predict the

27 component parts of the virions with a confidence level well above other computational tools. The

$28 \mathrm{STEP}^{3}$ toolbox is available at http://step3.erc.monash.edu/. 


\section{METHODS}

\section{Construction of the Klebsiella host strain}

3 B5055 is a multidrug-resistant $K$. pneumoniae $(40,41)$ strain with a K2-type capsule considered

4 indicative of hypervirulent K. pneumoniae (hvKp) (42). To avoid isolating phages that use the

5 major porin for entry into K. pneumoniae (33) and, thus circumvent the prospect of phage-

6 resistance acquired by decreased expression of porins (43) and collateral increases in drug-

7 resistant phenotype in the infection (44), we constructed as bait a strain that has no OmpK36.

8 This $\triangle$ ompK36 mutant strain of $K$. pneumoniae B5055 was constructed by "gene gorging" as

9 previously described $(45,46)$ utilizing the donor and helper plasmids described in

10 Supplementary Table 10.

\section{Phage isolation and infection of Klebsiella}

13 Water samples were collected from catchment locations along the Merri Creek in Melbourne,

14 Australia (Reservoir, postcode 3073, yielded MMNM, and Pascoe Vale, postcode 3044, yielded

15 MMBB). Samples were centrifuged at $10,000 \times g$ for 10 minutes and filtered through a $0.45 \mu \mathrm{m}$

16 cut-off filter. Water samples $(45 \mathrm{~mL})$ were subsequently mixed with $5 \mathrm{~mL}$ of $10 \times$ concentrated

17 Luria-Bertani (LB) media and $1 \mathrm{~mL}$ of a K. pneumoniae B5055 $\Delta$ ompK36 overnight culture and

18 grown for a further 16 hours at $37^{\circ} \mathrm{C}$. Cellular debris were pelleted by centrifugation at $10,000 \times$

$19 g$ for 10 minutes and the resulting supernatant was passed through a $0.45 \mu \mathrm{m}$ filter. To monitor

20 phage activity, $20 \mu \mathrm{L}$ of the supernatant was then spotted onto LB agar plates containing a top

21 layer of soft agar (4 mL LB and $0.35 \%$ (w/v) agar) and $200 \mu \mathrm{L}$ of bacterial culture and incubated

22 overnight at $37^{\circ} \mathrm{C}$.

24 For liquid infections, the filtered supernatant was serially diluted with $\mathrm{SM}$ buffer $(100 \mathrm{mM} \mathrm{NaCl}$,

$258 \mathrm{mM} \mathrm{MgSO}_{4}, 10 \mathrm{mM}$ Tris $\mathrm{pH} 7.5$ ) and added to $200 \mu \mathrm{L}$ of $K$. pneumoniae B5055 $\Delta$ ompK36.

26 Cultures were incubated for 20 minutes at $37^{\circ} \mathrm{C}$ to allow phage adsorption and were then added

27 to soft agar and poured using the double overlay method. Plaques with distinct morphologies

28 were isolated from the top agar, serially diluted in SM buffer and incubated with the bacterial

29 host as described above. This was repeated 5 times to obtain pure phage stocks.

\section{$31 \quad$ Phage amplification and purification}

32 For large -amplification of the phages MMNM and MMBB, infections were performed using 14

$33 \mathrm{~cm}$ petri dishes with $60 \mu \mathrm{L}$ of phage preparation $\left(10^{-4}\right.$ dilution) added to $500 \mu \mathrm{L}$ of an overnight

34 culture and incubated for 20 minutes at $37^{\circ} \mathrm{C}$. Ten millilitres of soft agar was then added to the

35 culture and poured using the double agar layer method and incubated overnight at $37^{\circ} \mathrm{C}$. Ten 
1 millilitres of SM buffer were added to each plate and incubated at room temperature for 10

2 minutes. The soft agar layer was scraped off using a disposable spreader and chloroform was

3 subsequently added ( $1 \mathrm{~mL} / 100 \mathrm{~mL})$ to lyse bacterial cells to release the phages. The sample was

4 then subject to vigorous shaking, before the agar and bacterial cell debris were removed by

5 centrifugation at $11,000 \times g$ for 40 minutes $\left(4^{\circ} \mathrm{C}\right)$. The supernatant containing the phages was

6 collected and DNase $(1 \mu \mathrm{g} / \mathrm{mL})$ and RNase $(1 \mu \mathrm{g} / \mathrm{mL})$ were subsequently added to the

7 supernatant and incubated for 30 minutes at $4^{\circ} \mathrm{C} . \mathrm{NaCl}(1 \mathrm{M}$ final concentration) was added and

8 incubated at $4^{\circ} \mathrm{C}$ for 1 hour with gentle mixing. Phages were precipitated from the media by

9 adding PEG 8000 (10\% final concentration) and incubated at $4^{\circ} \mathrm{C}$ overnight. Precipitated phage

10 particles were collected by centrifugation at $11,000 \times g$ for 20 minutes at $4^{\circ} \mathrm{C}$ and resuspended in

$11 \mathrm{SM}$ buffer $(1.6 \mathrm{~mL} / 100 \mathrm{~mL}$ of precipitated supernatant). An equal volume of chloroform was

12 added to the resuspended phage suspension to remove residual PEG and cell debris and vortexed

13 for 30 seconds. The organic and aqueous phases were separated by centrifugation at 3,000 $\times g$

14 for 15 minutes at $4^{\circ} \mathrm{C}$.

16 For purification on caesium chloride $(\mathrm{CsCl})$ gradients, the aqueous phase containing the phages

17 was removed and added to $\mathrm{CsCl}(0.5 \mathrm{~g} / \mathrm{mL}$ of bacteriophage suspension) and mixed gently to

18 dissolve the $\mathrm{CsCl}$. The suspension was layered onto a discontinuous $\mathrm{CsCl}$ gradient (2 $\mathrm{mL}$ of 1.70

$19 \mathrm{~g} / \mathrm{mL}, 1.5 \mathrm{~mL}$ of $1.50 \mathrm{~g} / \mathrm{mL}$ and $1.5 \mathrm{~mL}$ of $1.45 \mathrm{~g} / \mathrm{mL}$ in $\mathrm{SM}$ buffer) in a Beckman SW41

20 centrifuge tube. Gradients were centrifuged at $22,000 \mathrm{rpm}$ for 2 hours $\left(4^{\circ} \mathrm{C}\right)$. Phage particles

21 were collected from the gradient by piercing the side of the centrifuge tube with a syringe and

22 removing the visible band in the gradient. Residual nucleic acid was removed from the phage

23 preparation using floatation gradient centrifugation. Equal volumes of phage suspension (500

$24 \mu \mathrm{L}$ ) and 7.2 M CsCl SM buffer were mixed and added to the bottom of a Beckman SW41

25 centrifuge tube. $\mathrm{CsCl}$ solutions ( $3 \mathrm{~mL}$ of $5 \mathrm{M}$ and $7.5 \mathrm{~mL}$ of $3 \mathrm{M}$ ) were overlaid on top of the

26 phage sample and centrifuged at 22,000 rpm for 2 hours $\left(4^{\circ} \mathrm{C}\right)$. Phage particles were collected

$27(\sim 500 \mu \mathrm{L})$ using a syringe as described above. $\mathrm{CsCl}$ was dialysed out of the phage stock twice

28 with $2 \mathrm{~L}$ of SM buffer overnight at $4{ }^{\circ} \mathrm{C}$.

\section{Phage growth}

31 One-step growth curve experiments were performed on $K$. pneumoniae as previously described

32 (29). Mid-log-phase cultures were adjusted to an optical density at $600 \mathrm{~nm}\left(\mathrm{OD}_{600}\right)$ of 0.5 ,

33 pelleted, and suspended in 0.1 volume of SM buffer. Phage lysate was subsequently added at a

34 multiplicity of infection (MOI) of 0.01 and was allowed to adsorb for 10 minutes at $37^{\circ} \mathrm{C}$.

35 Following centrifugation at $12,000 \times g$ for 4 minutes, the pellet was washed twice with SM

36 buffer, resuspended with $30 \mathrm{~mL}$ of fresh $\mathrm{LB}$ broth, and incubated at $37^{\circ} \mathrm{C}$. Samples were 
1 collected at 10-minute intervals for 120 minutes and titrated to determine PFU/mL. Growth

experiments were performed in biological triplicates.

\section{Electron microscopy}

5 From the CsCl-purifications, phage preparations ( $4 \mu \mathrm{L})$ were added to freshly glow-discharged

6 CF200-Cu Carbon Support Film 200 Mesh Copper grids (ProScieTech) for 30 seconds. The

7 sample was blotted from the grid using Whatman filter paper and samples were subsequently

8 stained with $4 \mu \mathrm{L}$ of Nano W Methylamine Tungstate (Nanoprobes) for 30 seconds and blotted

9 again. Grids were imaged using a 120keV Tecnai Spirit G2 transmission electron microscope

10 (Tecnai).

\section{Genomic DNA extraction, sequencing and annotation}

13 Phage genomic DNA was isolated and samples were sequenced as $2 \times 250 \mathrm{bp}$ paired-end reads 14 using Illumina MiSeq (36). The obtained reads were trimmed using Trimmomatic (47) and de

15 novo assemblies of each genome were made using Burrows-Wheeler aligner (48) and Spades

16 (49). The genomes were annotated using Prokka (50). The consensus sequences were then

17 screened against the GenBank database using BLAST (https://blast.ncbi.nlm.nih.gov/Blast.cgi),

18 date 29 April 2020. The genome data is available at Genbank with Accession ID:

19 Klebsiella_phage_MMNM (MT894004) and Klebsiella_phage_MMBB (MT894005).

\section{Comparative genome analyses and BLAST}

22 Proteomic trees were constructed using nucleotide genome sequences using the double stranded

23 (ds) DNA nucleic acid type and Prokaryote host category database from ViPTree v1.9 (51)

24 which also included a list of curated phage genomes (Supplementary Table 11). Refined trees

25 were regenerated to analyse the phylogeny of either Myoviridae or Siphoviridae that infect

26 Gammaproteobacteria. Each predicted open reading frame was analysed using BLASTP

27 (https://blast.ncbi.nlm.nih.gov/Blast.cgi), Pfam HMMER (https://www.ebi.ac.uk/Tools/hmmer/)

28 and HHpred (https://toolkit.tuebingen.mpg.de/) using the default settings.

30 A BLAST-based predictor was implemented during the evaluation of STEP ${ }^{3}$. It ran using blast-

$312.2 .26+$ For a query protein, the BLAST-based predictor will predict it to be positive if there is a

32 BLAST hit against the training positive samples with a specified E-value. The E-value was set to

330.01 in this study, optimized on the independent dataset with a range of: $0.001,0.01,0.1,1$, and

3410. 
1 Each $\mathrm{CsCl}$ purified phage sample was solubilized in sodium dodecyl sulfate (SDS) lysis buffer

2 (4\% SDS, $100 \mathrm{mM}$ HEPES pH8.5) and sonicated to assist protein extraction. The protein

3 concentration was determined using a BCA kit (Thermo Scientific). SDS was removed according

4 to previous work (52) and the proteins were proteolytically digested with trypsin (Promega) and

5 purified using OMIX C18 Mini-Bed tips (Agilent Technologies) prior to LC-MS/MS analysis.

6 Using a Dionex UltiMate 3000 RSLCnano system equipped with a Dionex UltiMate 3000 RS

7 autosampler, an Acclaim PepMap RSLC analytical column $(75 \mu \mathrm{m} \times 50 \mathrm{~cm}$, nanoViper, C18, 2

$8 \mu \mathrm{m}, 100 \AA$; Thermo Scientific) and an Acclaim PepMap 100 trap column $(100 \mu \mathrm{m} \times 2 \mathrm{~cm}$,

9 nanoViper, $\mathrm{C} 18,5 \mu \mathrm{m}, 100 \AA$; Thermo Scientific), the tryptic peptides were separated by

10 increasing concentrations of $80 \%$ acetonitrile $/ 0.1 \%$ formic acid at a flow of $250 \mathrm{~nL} / \mathrm{min}$ for 120

11 minutes and analyzed with a QExactive Plus mass spectrometer (Thermo Scientific) using in-

12 house optimized parameters to maximize the number of peptide identifications. To obtain

13 peptide sequence information, the raw files were searched with Byonic v3.0.0 (ProteinMetrics)

14 against the $K$. pneumoniae B5055 GenBank file FO834906 that was appended with the phage

15 protein sequences. Only proteins falling within a false discovery rate (FDR) of $1 \%$ based on a

16 decoy database were considered for further analysis.

\section{Raw data availability}

19 The mass spectrometry proteomics data have been deposited to the ProteomeXchange

20 Consortium via the PRIDE (53) partner repository with the dataset identifier PXD020607.

21 Username: reviewer30311@ebi.ac.uk, Password: ggYKM6wi

\section{Homology Modelling}

24 Structural homologues were selected by querying the MMBB_78 sequence via the BLASTp webserver against the Protein Databank (PDB). In addition, this same sequence was probed using the Phyre2 software suite to identify local homology (54). Residues 186-872 of MMBB_78 were modelled against the enzymatic domain of the bacteriophage CBA120 tailspike protein (PDB ID: 5W6P (55)). MODELLER v9.19 (56) was used with custom in-house scripts to generate 1000 potential models. These models were validated and sorted by their Discrete Optimised Protein Energy (DOPE) score, followed by visual inspection. An additional atomic model was calculated by the predictive software GalaxyTBM using the full length MMBB_78 sequence, as part of the GalaxyWEB (57) software suite.

\section{Construction of $S T E P^{3}$}

35 Dataset construction. 481 phage virion proteins were collected from the UniProt database with

36 the "reviewed" tag and from the NCBI database following extensive literature searches. 
1 Redundant sequences were removed using the CD-HIT program (58) at a cut-off threshold of 0.4.

2 As a result, 339 virion proteins with less than $40 \%$ sequence similarity were obtained. These

3 proteins were further divided into two parts as positive samples: 243 in the training dataset and

496 in the independent dataset. For negative samples, 694 and 96 phage non-virion proteins were

5 collected from UniProt to make up the training and independent datasets, respectively. Finally, a

6 training dataset (243 positive samples and 694 negative samples) and an independent dataset (96

7 positive samples and 96 negative samples) were obtained, where each had less than $40 \%$

8 sequence similarity against each other. The two newly sequenced phage genomes MMNM and

$9 \mathrm{MMBB}$ in this study were used to validate the prediction capability of $\mathrm{STEP}^{3}$ in practical

10 scenarios.

11 PSSM generation. PSSM is a $\mathrm{L}^{*} 20$ matrix, where $\mathrm{L}$ is the length of its original protein sequence

12 and 20 is the number of amino acids. The (i,j)-th element $(1<=i<=L, 1<=j<=20)$ in a PSSM

13 corresponds to the probability of $\mathrm{j}$-th amino acid to appear in the $\mathrm{i}$-th position of its protein

14 sequence. To generate a PSSM, blast-2.2.26 resource

15 (ftp://ftp.ncbi.nlm.nih.gov/blast/executables) was used to search the protein sequence against the

16 UniRef50 dataset (https://www.uniprot.org/help/uniref) with an E-value of 0.001 and the

17 iteration of 3.

18 Feature encoding. Instead of extracting features directly from the protein sequences,

19 evolutionary features mine patterns from a more informative profile in the format of PSSM. Five

20 types of evolutionary features were generated using the POSSUM toolkit (59), including AAC-

21 PSSM (60), PSSM-composition (61), DPC-PSSM (60), AADP-PSSM (60), and MEDP (62). For

22 a given PSSM, their calculations are briefly described as follows: 1) AAC-PSSM generates a 20-

23 dimensional vector through summing up and averaging all rows of the PSSM (60). 2) PSSMcomposition further divides PSSM rows into 20 groups according to their corresponding amino acids in the original protein sequence (61). The rows in each group are summed up and normalized, and as a result the PSSM are transformed into a $20 * 20$ matrix. Converting this matrix into a vector by row, PSSM-composition finally generates a 400-dimensional vector. 3) DPC-PSSM generates a 400-dimensional vector $\left(y_{1,1}, \ldots, y_{1,20}, y_{2,1}, \ldots, y_{2,20}, \ldots, y_{20,1}, \ldots, y_{20,20}\right)^{T}$ through taking into account the local sequence-order effect (60). Among the vector, $y_{i, j}$ can be calculated by $\frac{1}{L-1} \sum_{k=1}^{L-1} p_{k, i} \times p_{k+1, j}$ where $\mathrm{i}$ and $\mathrm{j}$ are between 1 and 20 , and $p_{k, i}$ denotes the $(\mathrm{k}, \mathrm{i})$-th element in PSSM. 4) AADP-PSSM combines AAC-PSSM and DPC-PSSM (60) as a 420-dimensional vector. 5) Likewise, MEDP generates a 420-dimensional vector through combining another two features EEDP and EDP (62). Among them, EEDP generates a 400dimensional vector similarly to DPC-PSSM but using different transformation methodologies. 
1 EDP further sums up and averages all rows of the EEDP matrix to generate a 20-dimensional vector.

4 Additionally, four commonly used features were additionally implemented for comparison 5 purpose, including the amino acid composition (AAC), dipeptide composition (DPC), QSOrder

6 (63) and PAAC (64). AAC and DPC count the frequencies of residues and dipeptides in a protein

7 sequence, respectively. QSOrder and PAAC extract features from a protein sequence as well,

8 incorporating the physicochemical properties of its individual amino acids. Among them,

9 QSOrder adopts Schneider-Wrede physicochemical distance matrix (65) and Grantham's

10 distance matrix (66), while PAAC takes hydrophobicity value from Tanford (67) and from Hopp

11 and Woods (68), as well as amino acid side chain.

12 Model training on imbalanced data. Our imbalanced training dataset is to reflect the fact that the 13 number of virion proteins is usually smaller than that of the non-virion proteins in a phage isolate.

14 We combined all of the virion proteins with the same number of randomly selected non-virion

15 proteins to generate a new balanced subset. This procedure was repeated five times, to generate

16 five balanced subsets. For each feature, five individual models were trained based on five

17 balanced subsets, and their prediction scores were averaged to obtain an ensemble model as the

18 baseline model. Support vector machine (SVM) with a radial basis function kernel was used to

19 train each model, implemented by the e1071 package (https://CRAN.R-

20 project.org/package=e1071) in the R language (https://www.r-project.org/). The two parameters

21 of SVM, including the Cost and Gamma, were optimized by a grid search between $2^{-10}$ and $2^{10}$

22 with a step of $2^{1}$ using the same R package.

23 Model integration. Training a model with each of features and then integrating them as an 24 ensemble model usually have better and more robust performance, when compared with simply 25 training a model with all features (69). Accordingly, the five baseline models (corresponding to 26 five evolutionary features) were further integrated as the final ensemble model of STEP ${ }^{3}$ through 27 averaging their prediction scores (Fig 1a).

28 Performance evaluation. The $\mathrm{STEP}^{3}$ predictor was extensively validated, with the baseline 29 models and existing state-of-the-art tools on the 5-fold cross-validation and independent tests.

30 Five performance metrics were used, including sensitivity (SN), specificity (SP), accuracy

31 (ACC), F-value and Matthews correlation coefficient (MCC) (70). For each model, 5-fold cross32 validation tests were conducted 5 times based on the 5 balanced training datasets, and then the 33 performance metrics were averaged as the final performance result. The other tools compared to 34 STEP $^{3}$ were iVIREONS (https://vdm.sdsu.edu/ivireons), PVPred (http://lin- 
1 group.cn/server/PVPred), PVP-SVM (http://www.thegleelab.org/PVP-SVM/PVP-SVM.html),

2 PVPred-SCM (http://camt.pythonanywhere.com/PVPred-SCM) and Pred-BVP-Unb (21). With

3 no available tool for Pred-BVP-Unb, we developed one based on our training dataset by strictly

4 following its methods, including its synthetic minority oversampling technique (SMOTE) to

5 cope with the imbalance dataset, feature encodings, feature selection (a more geneneralized

6 method GainRatio used) and the same grid search for parameter optimization. The prediction

7 threshold for Pred-BVP-Unb is a standard cut-off of 0.5 , which is the same as STEP ${ }^{3}$.

9 Sever construction and usage. The $\mathrm{STEP}^{3}$ server contains a client web interface and a server

10 backend. The client web interface was implemented by the JAVA (https://www.java.com/)

11 server development suite, JSP, CSS, jQuery (https://jquery.com/), Bootstrap

12 (https://bootstrapdocs.com/) and their extension packages. The server backend was used by the

13 Perl CGI (https://metacpan.org/pod/CGI). For visualization purposes, the blast 2.8.1+

14 (ftp://ftp.ncbi.nlm.nih.gov/blast/executables/blast+/2.8.1/) was used to search each predicted

15 virion protein against known virion proteins to generate sequence similarities, which was

16 visualized by BlasterJS (71). The MAFFT v7.271 (https://mafft.cbrc.jp/alignment/software/) was

17 used to generate multiple alignment results between each predicted virion protein and known

18 virion proteins, which was visualized by jsPhyloSVG (72). The all-against-all BLAST (version

19 blast-2.2.26) was used to generate the sequence similarity network, visualized by ECharts

20 (https://echarts.apache.org/). A queuing system was implemented using the Gearman framework

21 (http://gearman.org/) to store the jobs the client deposits and dispatch them to idle threads

22 maintained in the server backend. In this way, it links the two parts of STEP ${ }^{3}$, but decouples the

23 prompt-response required in a client web interface and the time-consuming server backend for

24 better user experience. To use the STEP $^{3}$ server, users submit their protein sequences in FASTA

25 format, and obtain a unique link to track the prediction progress or obtain the results once

26 finished. In default mode, i.e. 'For normal use', the known virion proteins were marked with

27 'exp.' with an external link to the UniProt or NCBI database, while the predicted virion proteins

28 were marked with 'pred.' with detailed annotations and options for visualization. Through

29 interactive visualization, users could tentatively annotate the putative virion proteins with their

30 potential subtype or functions, based on the sequence similarity or phylogenetic analysis

31 considerations. For users who want to benchmark the STEP ${ }^{3}$ server, a 'For benchmarking test'

32 option is available to obtain prediction scores for all their sequences. 


\section{REFERENCES}

2 1. Control CfD, Prevention. 2019. Antibiotic resistance threats in the United States, 2019.

3 Atlanta, GA: US Department of Health and Human Services, Centers for Disease Control

$4 \quad$ and Prevention; 2019.

$5 \quad 2 . \quad$ O'Neill J. 2019. Tackling drug-resistant infections globally: final report and recommendations: the review on antimicrobial resistance; 2016 [Available from: https://amr-review. org. Publications html.

3. Luong T, Salabarria AC, Edwards RA, Roach DR. 2020. Standardized bacteriophage purification for personalized phage therapy. Nat Protoc.

4. Gorski A, Miedzybrodzki R, Lobocka M, Glowacka-Rutkowska A, Bednarek A, Borysowski J, Jonczyk-Matysiak E, Lusiak-Szelachowska M, Weber-Dabrowska B, Baginska N, Letkiewicz S, Dabrowska K, Scheres J. 2018. Phage Therapy: What Have We Learned? Viruses 10.

5. Rohde C, Wittmann J, Kutter E. 2018. Bacteriophages: A Therapy Concept against Multi-Drug-Resistant Bacteria. Surg Infect (Larchmt) 19:737-744.

6. Pires DP, Costa AR, Pinto G, Meneses L, Azeredo J. 2020. Current challenges and future opportunities of phage therapy. FEMS Microbiol Rev.

7. McNair K, Aziz RK, Pusch GD, Overbeek R, Dutilh BE, Edwards R. 2018. Phage Genome Annotation Using the RAST Pipeline. Methods Mol Biol 1681:231-238.

8. McNair K, Zhou C, Dinsdale EA, Souza B, Edwards RA. 2019. PHANOTATE: a novel approach to gene identification in phage genomes. Bioinformatics 35:4537-4542.

9. Hardy JM, Dunstan RA, Grinter R, Belousoff MJ, Wang J, Pickard D, Venugopal H, Dougan G, Lithgow T, Coulibaly F. 2020. The architecture and stabilisation of flagellotropic tailed bacteriophages. Nat Commun 11:3748.

10. Fokine A, Rossmann MG. 2014. Molecular architecture of tailed double-stranded DNA phages. Bacteriophage 4:e28281.

11. Davidson AR, Cardarelli L, Pell LG, Radford DR, Maxwell KL. 2012. Long noncontractile tail machines of bacteriophages. Adv Exp Med Biol 726:115-42.

12. Leiman PG, Shneider MM. 2012. Contractile tail machines of bacteriophages. Adv Exp Med Biol 726:93-114.

13. Fernandes S, Sao-Jose C. 2018. Enzymes and Mechanisms Employed by Tailed Bacteriophages to Breach the Bacterial Cell Barriers. Viruses 10.

14. Salmond GP, Fineran PC. 2015. A century of the phage: past, present and future. Nat Rev Microbiol 13:777-86.

15. Ecale Zhou CL, Malfatti S, Kimbrel J, Philipson C, McNair K, Hamilton T, Edwards R, Souza B. 2019. multiPhATE: bioinformatics pipeline for functional annotation of phage isolates. Bioinformatics 35:4402-4404.

16. Mavrich TN, Hatfull GF. 2017. Bacteriophage evolution differs by host, lifestyle and genome. Nat Microbiol 2:17112.

17. Seguritan V, Alves N, Jr., Arnoult M, Raymond A, Lorimer D, Burgin AB, Jr., Salamon P, Segall AM. 2012. Artificial neural networks trained to detect viral and phage structural proteins. PLoS Comput Biol 8:e1002657.

18. Ding H, Feng PM, Chen W, Lin H. 2014. Identification of bacteriophage virion proteins by the ANOVA feature selection and analysis. Mol Biosyst 10:2229-35.

19. Manavalan B, Shin TH, Lee G. 2018. PVP-SVM: Sequence-Based Prediction of Phage Virion Proteins Using a Support Vector Machine. Front Microbiol 9:476.

20. Pan Y, Gao H, Lin H, Liu Z, Tang L, Li S. 2018. Identification of Bacteriophage Virion Proteins Using Multinomial Naive Bayes with g-Gap Feature Tree. Int J Mol Sci 19.

21. Arif M, Ali F, Ahmad S, Kabir M, Ali Z, Hayat M. 2020. Pred-BVP-Unb: Fast prediction of bacteriophage Virion proteins using un-biased multi-perspective properties with recursive feature elimination. Genomics 112:1565-1574. 
22. Charoenkwan P, Kanthawong S, Schaduangrat N, Yana J, Shoombuatong W. 2020. PVPred-SCM: Improved Prediction and Analysis of Phage Virion Proteins Using a Scoring Card Method. Cells 9.

23. Meng C, Zhang J, Ye X, Guo F, Zou Q. 2020. Review and comparative analysis of machine learning-based phage virion protein identification methods. Biochim Biophys Acta Proteins Proteom 1868:140406.

24. Jeong JC, Lin X, Chen XW. 2011. On position-specific scoring matrix for protein function prediction. IEEE/ACM Trans Comput Biol Bioinform 8:308-15.

25. Wang J, Dai W, Li J, Xie R, Dunstan RA, Stubenrauch C, Zhang Y, Lithgow T. 2020. PaCRISPR: a server for predicting and visualizing anti-CRISPR proteins. Nucleic Acids Res 48:W348-W357.

26. Topka-Bielecka G, Bloch S, Nejman-Falenczyk B, Grabski M, Jurczak-Kurek A, Gorniak M, Dydecka A, Necel A, Wegrzyn G, Wegrzyn A. 2020. Characterization of the Bacteriophage vB_EfaS-271 Infecting Enterococcus faecalis. Int J Mol Sci 21.

27. Buttimer C, Lynch C, Hendrix H, Neve H, Noben JP, Lavigne R, Coffey A. 2020. Isolation and Characterization of Pectobacterium Phage vB_PatM_CB7: New Insights into the Genus Certrevirus. Antibiotics (Basel) 9.

28. Necel A, Bloch S, Nejman-Falenczyk B, Grabski M, Topka G, Dydecka A, KosznikKwasnicka K, Grabowski L, Jurczak-Kurek A, Wolkowicz T, Wegrzyn G, Wegrzyn A. 2020. Characterization of a bacteriophage, vB_Eco4M-7, that effectively infects many Escherichia coli O157 strains. Sci Rep 10:3743.

29. D'Andrea MM, Marmo P, Henrici De Angelis L, Palmieri M, Ciacci N, Di Lallo G, Dematte E, Vannuccini E, Lupetti P, Rossolini GM, Thaller MC. 2017. phiBO1E, a newly discovered lytic bacteriophage targeting carbapenemase-producing Klebsiella pneumoniae of the pandemic Clonal Group 258 clade II lineage. Sci Rep 7:2614.

30. Hung $\mathrm{CH}$, Kuo CF, Wang CH, Wu CM, Tsao N. 2011. Experimental phage therapy in treating Klebsiella pneumoniae-mediated liver abscesses and bacteremia in mice. Antimicrob Agents Chemother 55:1358-65.

31. Arisaka F, Yap ML, Kanamaru S, Rossmann MG. 2016. Molecular assembly and structure of the bacteriophage T4 tail. Biophys Rev 8:385-396.

32. Yap ML, Klose T, Arisaka F, Speir JA, Veesler D, Fokine A, Rossmann MG. 2016. Role of bacteriophage T4 baseplate in regulating assembly and infection. Proc Natl Acad Sci U S A 113:2654-9.

33. Cai R, Wu M, Zhang H, Zhang Y, Cheng M, Guo Z, Ji Y, Xi H, Wang X, Xue Y, Sun C, Feng X, Lei L, Tong Y, Liu X, Han W, Gu J. 2018. A Smooth-Type, Phage-Resistant Klebsiella pneumoniae Mutant Strain Reveals that OmpC Is Indispensable for Infection by Phage GH-K3. Appl Environ Microbiol 84.

34. Taylor NMI, van Raaij MJ, Leiman PG. 2018. Contractile injection systems of bacteriophages and related systems. Mol Microbiol 108:6-15.

35. Stverakova D, Sedo O, Benesik M, Zdrahal Z, Doskar J, Pantucek R. 2018. Rapid Identification of Intact Staphylococcal Bacteriophages Using Matrix-Assisted Laser Desorption Ionization-Time-of-Flight Mass Spectrometry. Viruses 10.

36. Dunstan RA, Pickard D, Dougan S, Goulding D, Cormie C, Hardy J, Li F, Grinter R, Harcourt K, Yu L, Song J, Schreiber F, Choudhary J, Clare S, Coulibaly F, Strugnell RA, Dougan G, Lithgow T. 2019. The flagellotropic bacteriophage YSD1 targets Salmonella Typhi with a Chi-like protein tail fibre. Mol Microbiol 112:1831-1846.

37. Manning KA, Dokland T. 2020. The gp44 Ejection Protein of Staphylococcus aureus Bacteriophage 80alpha Binds to the Ends of the Genome and Protects It from Degradation. Viruses 12.

38. Knecht LE, Veljkovic M, Fieseler L. 2019. Diversity and Function of Phage Encoded Depolymerases. Front Microbiol 10:2949.

39. Hendrix RW, Smith MC, Burns RN, Ford ME, Hatfull GF. 1999. Evolutionary relationships among diverse bacteriophages and prophages: all the world's a phage. Proc Natl Acad Sci U S A 96:2192-7. 
40. Paczosa MK, Mecsas J. 2016. Klebsiella pneumoniae: Going on the Offense with a Strong Defense. Microbiol Mol Biol Rev 80:629-61.

41. Kumari S, Harjai K, Chhibber S. 2011. Bacteriophage versus antimicrobial agents for the treatment of murine burn wound infection caused by Klebsiella pneumoniae B5055. J Med Microbiol 60:205-210.

42. Yeh KM, Kurup A, Siu LK, Koh YL, Fung CP, Lin JC, Chen TL, Chang FY, Koh TH. 2007. Capsular serotype $\mathrm{K} 1$ or $\mathrm{K} 2$, rather than magA and $\mathrm{rmpA}$, is a major virulence determinant for Klebsiella pneumoniae liver abscess in Singapore and Taiwan. J Clin Microbiol 45:466-71.

43. Tsai YK, Fung CP, Lin JC, Chen JH, Chang FY, Chen TL, Siu LK. 2011. Klebsiella pneumoniae outer membrane porins OmpK35 and OmpK36 play roles in both antimicrobial resistance and virulence. Antimicrob Agents Chemother 55:1485-93.

44. Rocker A, Lacey JA, Belousoff MJ, Wilksch JJ, Strugnell RA, Davies MR, Lithgow T. 2020. Global Trends in Proteome Remodeling of the Outer Membrane Modulate Antimicrobial Permeability in Klebsiella pneumoniae. mBio 11.

45. Wilksch JJ, Yang J, Clements A, Gabbe JL, Short KR, Cao H, Cavaliere R, James CE, Whitchurch CB, Schembri MA, Chuah ML, Liang ZX, Wijburg OL, Jenney AW, Lithgow T, Strugnell RA. 2011. MrkH, a novel c-di-GMP-dependent transcriptional activator, controls Klebsiella pneumoniae biofilm formation by regulating type 3 fimbriae expression. PLoS Pathog 7:e1002204.

46. Herring CD, Glasner JD, Blattner FR. 2003. Gene replacement without selection: regulated suppression of amber mutations in Escherichia coli. Gene 311:153-63.

47. Bolger AM, Lohse M, Usadel B. 2014. Trimmomatic: a flexible trimmer for Illumina sequence data. Bioinformatics 30:2114-20.

48. Li H, Durbin R. 2009. Fast and accurate short read alignment with Burrows-Wheeler transform. Bioinformatics 25:1754-60.

49. Bankevich A, Nurk S, Antipov D, Gurevich AA, Dvorkin M, Kulikov AS, Lesin VM, Nikolenko SI, Pham S, Prjibelski AD, Pyshkin AV, Sirotkin AV, Vyahhi N, Tesler G, Alekseyev MA, Pevzner PA. 2012. SPAdes: a new genome assembly algorithm and its applications to single-cell sequencing. J Comput Biol 19:455-77.

50. Seemann T. 2014. Prokka: rapid prokaryotic genome annotation. Bioinformatics 30:2068-9.

51. Nishimura Y, Yoshida T, Kuronishi M, Uehara H, Ogata H, Goto S. 2017. ViPTree: the viral proteomic tree server. Bioinformatics 33:2379-2380.

52. Zougman A, Selby PJ, Banks RE. 2014. Suspension trapping (STrap) sample preparation method for bottom-up proteomics analysis. Proteomics 14:1006-0.

53. Perez-Riverol Y, Csordas A, Bai J, Bernal-Llinares M, Hewapathirana S, Kundu DJ, Inuganti A, Griss J, Mayer G, Eisenacher M, Perez E, Uszkoreit J, Pfeuffer J, Sachsenberg T, Yilmaz S, Tiwary S, Cox J, Audain E, Walzer M, Jarnuczak AF, Ternent T, Brazma A, Vizcaino JA. 2019. The PRIDE database and related tools and resources in 2019: improving support for quantification data. Nucleic Acids Res 47:D442-D450.

54. Kelley LA, Mezulis S, Yates CM, Wass MN, Sternberg MJ. 2015. The Phyre2 web portal for protein modeling, prediction and analysis. Nat Protoc 10:845-58.

55. Plattner M, Shneider MM, Arbatsky NP, Shashkov AS, Chizhov AO, Nazarov S, Prokhorov NS, Taylor NMI, Buth SA, Gambino M, Gencay YE, Brondsted L, Kutter EM, Knirel YA, Leiman PG. 2019. Structure and Function of the Branched Receptor-Binding Complex of Bacteriophage CBA120. J Mol Biol 431:3718-3739.

56. Sali A, Blundell TL. 1993. Comparative protein modelling by satisfaction of spatial restraints. J Mol Biol 234:779-815.

57. Ko J, Park H, Heo L, Seok C. 2012. GalaxyWEB server for protein structure prediction and refinement. Nucleic Acids Res 40:W294-7.

58. Huang Y, Niu B, Gao Y, Fu L, Li W. 2010. CD-HIT Suite: a web server for clustering and comparing biological sequences. Bioinformatics 26:680-2. 
59. Wang J, Yang B, Revote J, Leier A, Marquez-Lago TT, Webb G, Song J, Chou KC, Lithgow T. 2017. POSSUM: a bioinformatics toolkit for generating numerical sequence feature descriptors based on PSSM profiles. Bioinformatics 33:2756-2758.

60. Liu T, Zheng X, Wang J. 2010. Prediction of protein structural class for low-similarity sequences using support vector machine and PSI-BLAST profile. Biochimie 92:1330-4.

61. Zou L, Nan C, Hu F. 2013. Accurate prediction of bacterial type IV secreted effectors using amino acid composition and PSSM profiles. Bioinformatics 29:3135-42.

62. Zhang L, Zhao X, Kong L. 2014. Predict protein structural class for low-similarity sequences by evolutionary difference information into the general form of Chou's pseudo amino acid composition. J Theor Biol 355:105-10.

63. Chou KC. 2000. Prediction of protein subcellular locations by incorporating quasisequence-order effect. Biochem Biophys Res Commun 278:477-83.

64. Chou KC. 2001. Prediction of protein cellular attributes using pseudo-amino acid composition. Proteins 43:246-55.

65. Schneider G, Wrede P. 1994. The rational design of amino acid sequences by artificial neural networks and simulated molecular evolution: de novo design of an idealized leader peptidase cleavage site. Biophys J 66:335-44.

66. Grantham R. 1974. Amino acid difference formula to help explain protein evolution. Science 185:862-4.

67. Tanford C. 1962. Contribution of hydrophobic interactions to the stability of the globular conformation of proteins. Journal of the American Chemical Society 84:4240-4247.

68. Hopp TP, Woods KR. 1981. Prediction of protein antigenic determinants from amino acid sequences. Proc Natl Acad Sci U S A 78:3824-8.

69. Wang J, Yang B, An Y, Marquez-Lago T, Leier A, Wilksch J, Hong Q, Zhang Y, Hayashida M, Akutsu T, Webb GI, Strugnell RA, Song J, Lithgow T. 2019. Systematic analysis and prediction of type IV secreted effector proteins by machine learning approaches. Brief Bioinform 20:931-951.

70. Matthews BW. 1975. Comparison of the predicted and observed secondary structure of T4 phage lysozyme. Biochimica et Biophysica Acta (BBA)-Protein Structure 405:442451.

71. Blanco-Miguez A, Fdez-Riverola F, Sanchez B, Lourenco A. 2018. BlasterJS: A novel interactive JavaScript visualisation component for BLAST alignment results. PLoS One 13:e0205286.

72. Smits SA, Ouverney CC. 2010. jsPhyloSVG: a javascript library for visualizing interactive and vector-based phylogenetic trees on the web. PLoS One 5:e12267. 


\section{ACKNOWLEDGEMENTS}

2 We acknowledge that this project was conducted on the traditional homelands of the Wurundjeri

3 Woi wurrung people, with the phages isolated from waters of the Merri Creek, Melbourne,

4 Australia. The Centre to Impact AMR would like to acknowledge and thank Wurundjeri Woi

5 wurrung Elder, Aunty Gail Smith, who named the phages in this study in Woi wurrung language.

6 Merri-merri-uth nyilam marra-natj (MMNM) and Merri-merri baany-a bundha-natj (MMBB)

7 translate as "Dangerous Merri lurker" and "Merri water biter", respectively, in English. Our

8 future work in this field will be pursued according to a Memorandum of Understanding (MoU)

9 between the Monash Centre to Impact AMR and the Wurundjeri Woi wurrung Cultural Heritage

10 Aboriginal Corporation (https://www.wurundjeri.com.au/) the peak body representing the

11 Wurundjeri Woi wurung people. The MoU recognizes the Wurundjeri Woi wurrung as the

12 sovereign First People of their Country with distinct rights, and will ensure the equitable sharing

13 of resources including any commercial benefits realized from the development of Wurundjeri

14 Woi wurrung resources. We acknowledge Jordan Smith and Karmen Jobling of the Wurundjeri

15 Woi wurrung Cultural Heritage Aboriginal Corporation's Water Unit for their stewardship in

16 shaping the MoU between Wurundjeri Woi wurrung Cultural Heritage Aboriginal Corporation

17 and Monash Centre to Impact AMR. We are grateful to Professor Richard Strugnell, Department

18 of Microbiology and Immunology, University of Melbourne for access to his collection of

19 Klebsiella isolates. W.D. was a visiting MSc student at Monash University, supported by the

20 study abroad program for graduate student of Guilin University of Electronic Technology

21 (GDYX2019010). Research was supported by a seed grant from the Monash-Warwick Alliance

22 (to T.L., E.J. and S.McG) and the initial phase of the project was supported by the Australian

23 Research Council (FL130100038).

\section{AUTHOR CONTRIBUTIONS}

TTY, MEW and JJW performed biological experiments. WD and YZ performed computational

to the final version of the manuscript.

\section{COMPETING INTERESTS}

35 The authors declare no competing interests. 


\section{FIGURE LEGENDS}

3 Figure 1. Construction and workflow for $\mathbf{S T E P}^{3}$. (a) Graphic summarizing the construction 4 and prediction process of STEP $^{3}$. A set of experimentally validated virion proteins and non-

5 virion proteins was compiled and sequence data fed into five PSSM models, including AAC-

$6 \operatorname{PSSM}(60), \operatorname{PSSM}-c o m p o s i t i o n(61), \operatorname{DPC}-P S S M(60), \operatorname{AADP}-P S S M(60)$, and a MEDP(62)

7 model. The five individual models were trained based on five balanced subsets, and their

8 prediction scores were averaged to obtain an ensemble model. Finally, five baseline models

9 (corresponding to five evolutionary features) were further integrated as the final ensemble model

10 of STEP $^{3}$ through averaging their prediction scores. Support vector machine (SVM) with a radial

11 basis function kernel was used to train each model. This ultimately provides a prediction of a

12 "virion protein" which would be a structural component of the phage virion. (b) STEP3 data

13 visualization provides a means to document relationships between a protein of interest. The

14 example given is the protein component gpE from phage $\lambda$, which shows clear similarity to

15 major capsid proteins from other phages. Structural studies confirm that despite limited sequence

16 similarity, gpE is part of a family of major capsid proteins(9). Alternative visualization features

17 are available in $\operatorname{STEP}^{3}$ (Supplementary Fig. 1).

Figure 2. Prediction details from STEP $^{3}$ and other tools. (a) For phage vB_EfaS_271,

horizontal bars denote the number of virion and non-virion proteins. The bar chart counts the virion proteins correctly retrieved as true positives (TP), i.e. confirmed by mass spectrometry (26), and non-virion proteins mistakenly predicted as virion proteins (denoted by false positives, FP). (b) For each protein in the phage vB_EfaS_271 virion defined by mass spectrometry, a green circle represents a successful hit by a predictor. (c) For phage vB_PatM_CB7, the bar chart counts the virion proteins correctly retrieved as TP and non-virion proteins mistakenly predicted as FP. (d) Detailed predictions from $\mathrm{STEP}^{3}$ and other tools for vB_PatM_CB7 virion proteins defined by mass spectrometry (27). (e) For phage vB_Eco4M-7, the bar chart counts the virion proteins correctly retrieved as TP and non-virion proteins mistakenly predicted as FP. (f) Detailed predictions from STEP $^{3}$ and other tools for vB_PatM_CB7 virion proteins defined by mass spectrometry (28).

Figure 3. Comparative genome analysis of Klebsiella phage MMNM. (a) Proteomic tree analysis of Myoviridae that infect Gammaproteobacteria. The branch lengths represent genomic similarity based on normalised pairwise sequence similarity scores plotted on a logarithmic scale. The tree was constructed using sequences from the default ViPTree dataset and phage genomes listed in Table S13. Viral subfamilies or genera are highlighted in the coloured bars. 
1 Gray bars represent phages that are currently unclassified. All known members of the

2 Jedunavirus, including Klebsiella phage MMNM (*), are highlighted in red. (b) Whole genome

3 alignment of Klebsiella phage_MMNM, vB_KpnM_FZ14 and vB_KpnM_KpV52. Each genome

4 has been oriented to start with the gene encoding the putative tape measure protein. The

5 sequences are linked by colored bars highlighting sequence identity values as shown in the key.

7 Figure 4. Morphological characterization of phage MMNM and MMBB. (a) Plaque

8 morphology analysis was performed using the double overlay method. Phages MMNM and

9 MMBB were serially diluted with SM buffer and spotted onto LB agar plates containing a top

10 layer of soft agar and K. pneumoniae B5055 $\Delta$ ompK36. Plaque morphologies of MMNM and

11 MMBB were determined after overnight incubation at $37^{\circ} \mathrm{C}$. Scale bars represent $10 \mathrm{~mm}$. (b)

12 One-step growth curve of MMNM (left) and MMBB (right) was performed by co-incubation

13 with the host strain for $10 \mathrm{~min}$ at $37^{\circ} \mathrm{C}$ for phage adsorption, after which the mixture was

14 subjected to centrifugation to remove free phage particles. The resuspended cell-phage pellets

15 were incubated at $37^{\circ} \mathrm{C}$ and sampled at $10 \mathrm{~min}$ intervals for $120 \mathrm{~min}$. L, latent period; B, burst

16 size. Data points are the mean of $n=3$ biologically independent samples and the error bars are the

17 standard deviation. (c) Transmission electron micrographs of MMNM (left) and MMBB (right).

18 The scale bars represent $100 \mathrm{~nm}$. (d) Based on EM micrographs, illustrations of MMNM (left)

19 and MMBB (right) note the cognate features in Myoviridae and Syphoviridae with annotation.

Figure 5. Prediction details from STEP $^{3}$ and other tools applied to MMNM and MMBB. (a)

22 The statistics of the prediction results on MMNM. Horizontal bars on top describe the number of

23 virion and non-virion proteins in the phage isolates. The bar chart counts the virion proteins

24 correctly retrieved (denoted by true positives [TP], i.e. confirmed by mass spectrometry) and

25 non-virion proteins mistakenly predicted as virion proteins (denoted by false positives [FP]). (b)

26 Detailed predictions from $\mathrm{STEP}^{3}$ and other tools for MMNM the virion proteins defined by mass spectrometry. The green circles represent a successful hit by a predictor. The green stars denote the proteins that have not previously been identified in phages. The red stars denote those with activities that have been previously identified in phages, but not previously found as protein components of purified virions. (c) Prediction statistics for MMBB. (d) Detailed predictions

31 from $\mathrm{STEP}^{3}$ and other tools for MMBB virion proteins defined by mass spectrometry. 


\section{SUPPLEMENT}

Supplementary Figure 1. Sequence analysis and data visualization of the major capsid protein from $\lambda$ phage.

Supplementary Figure 2. Comparative genome analysis of Klebsiella phage MMBB.

Supplementary Figure 3. Structure informed analysis of Klebsiella phage MMBB virion components.

Supplementary Table 1. Prediction performance of $\operatorname{STEP}^{3}$ and baseline models on the 5fold cross-validation test.

Supplementary Table 2. Prediction performance of $\mathrm{STEP}^{3}$ and baseline models on the on the independent test.

Supplementary Table 3. Prediction performance of $\mathrm{STEP}^{3}$, other available predictors and the BLAST-based baseline predictor on the independent dataset.

Supplementary Table 4. Annotation of Klebsiella phage MMNM genome.

Supplementary Table 5. Annotation of Klebsiella phage MMBB genome.

Supplementary Table 6. Mass spectrometry and STEP $^{3}$ analysis of Klebsiella phage MMNM virions.

Supplementary Table 7. Detailed prediction of $\mathrm{STEP}^{3}$, other available predictors and the BLAST-based baseline predictor on the phage Klebsiella phage MMNM.

Supplementary Table 8. Detailed prediction of $\mathrm{STEP}^{3}$, other available predictors and the BLAST-based baseline predictor on the phage Klebsiella phage MMBB.

Supplementary Table 9. Mass spectrometry and STEP $^{3}$ analysis of Klebsiella phage MMBB virions.

Supplementary Table 10. Strains, plasmids and primers used in this study.

Supplementary Table 11. Genomes of phages used for tree analysis. 

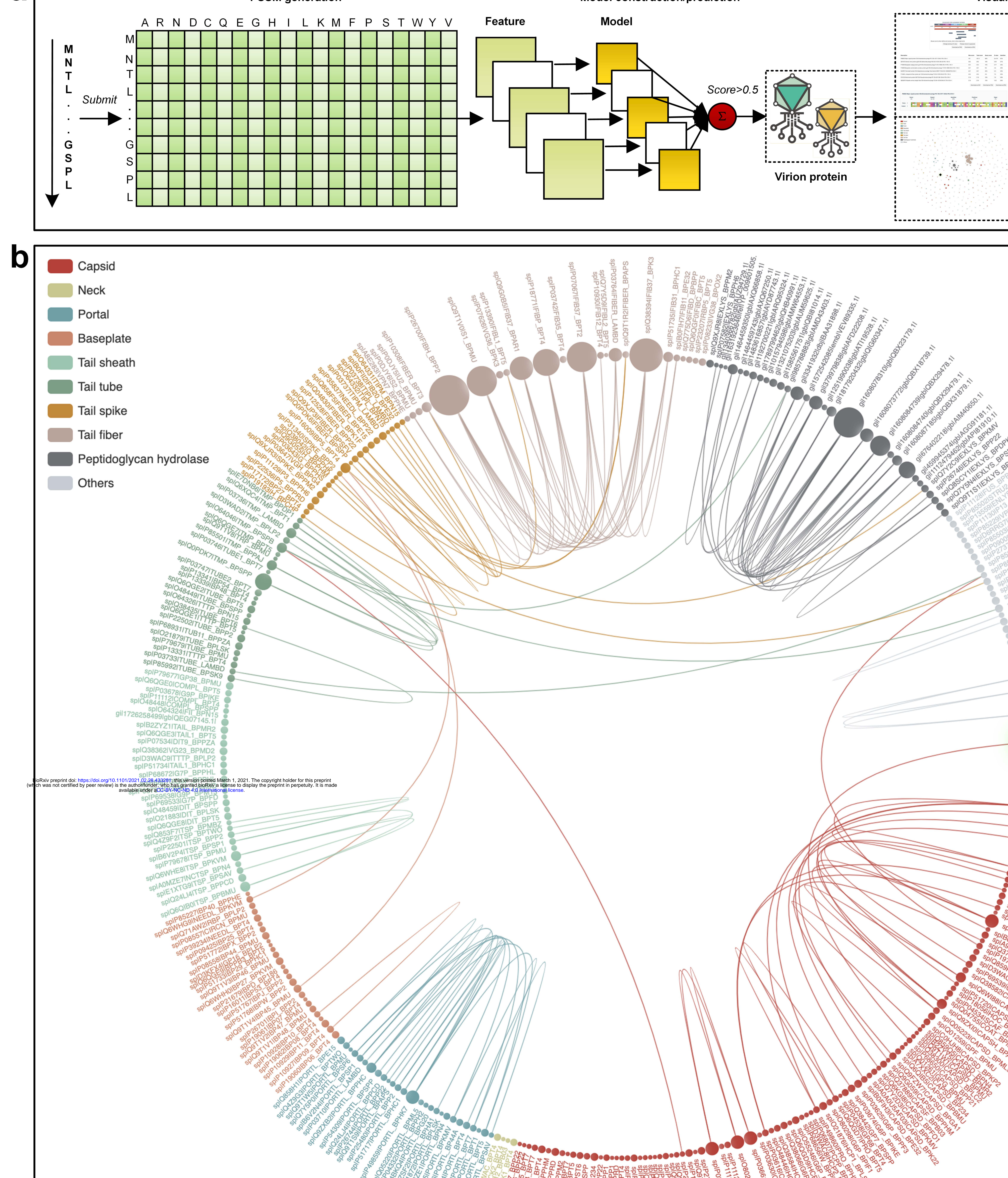

Others 
Phage: vB_EfaS_271

Host: Enterococcus faecalis

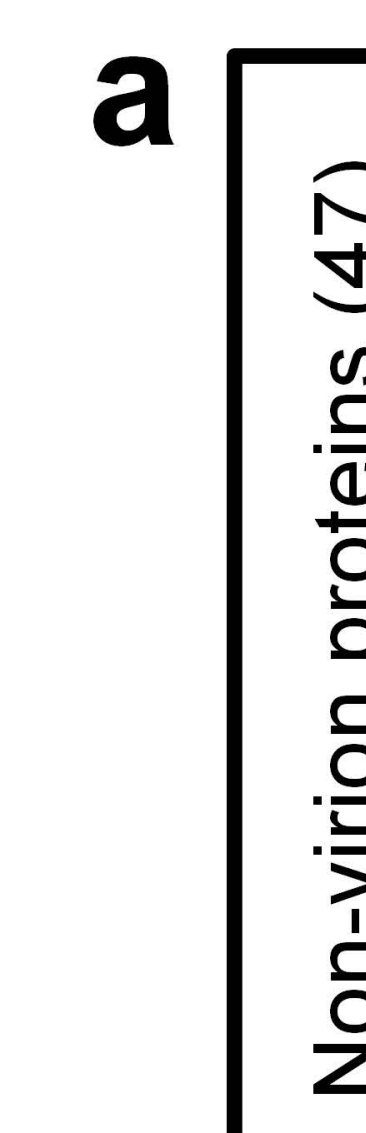

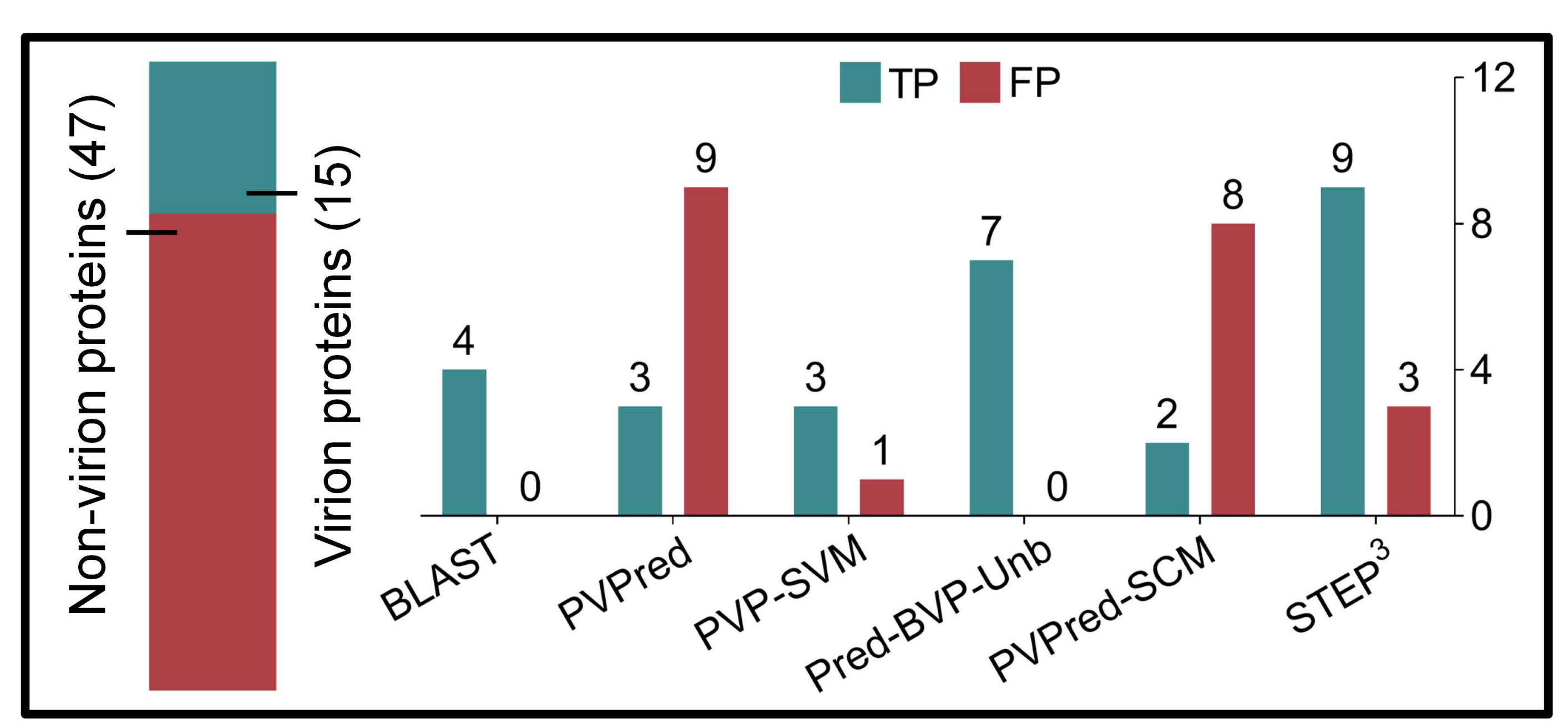

b

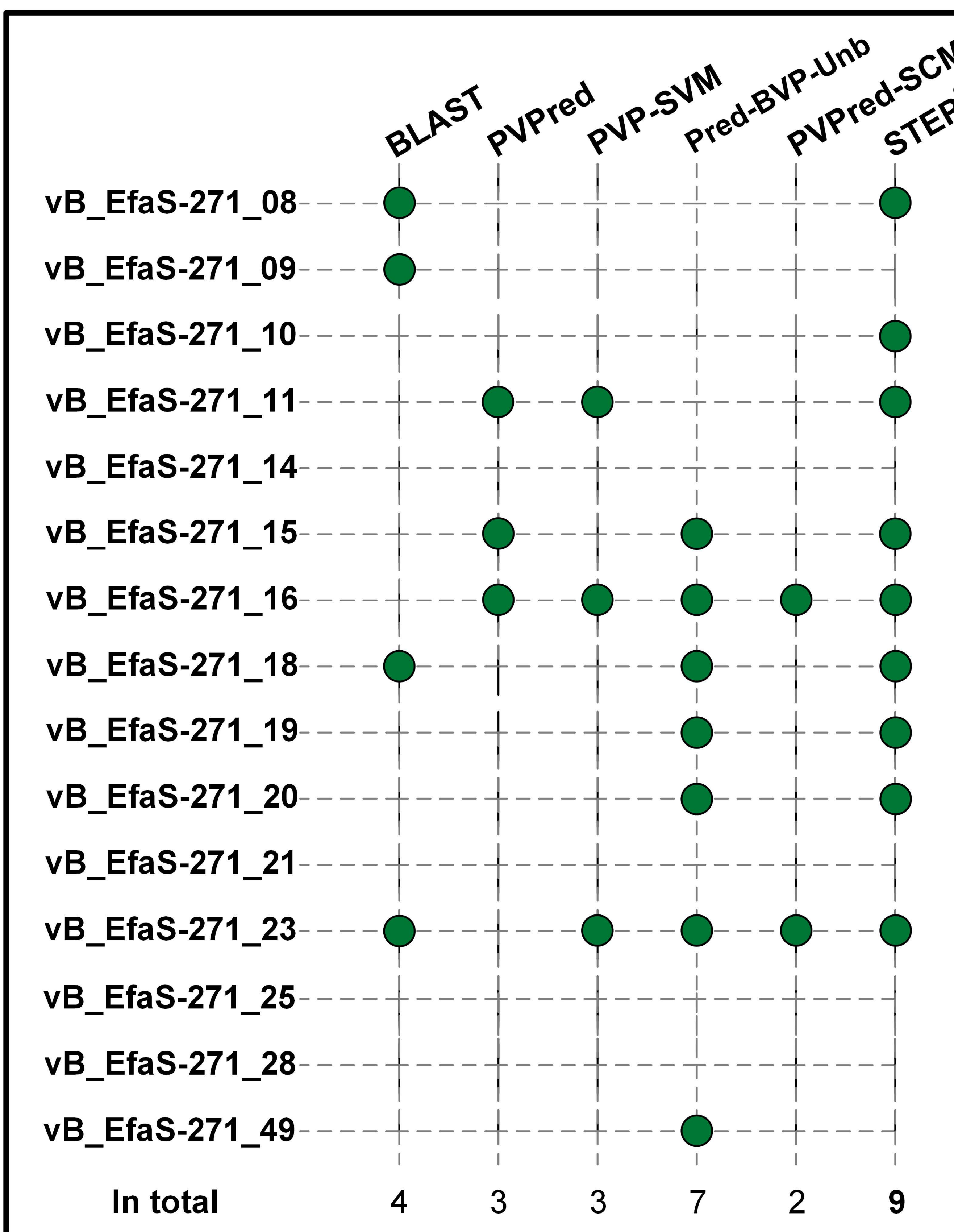

Phage: vB PatM CB7 oj

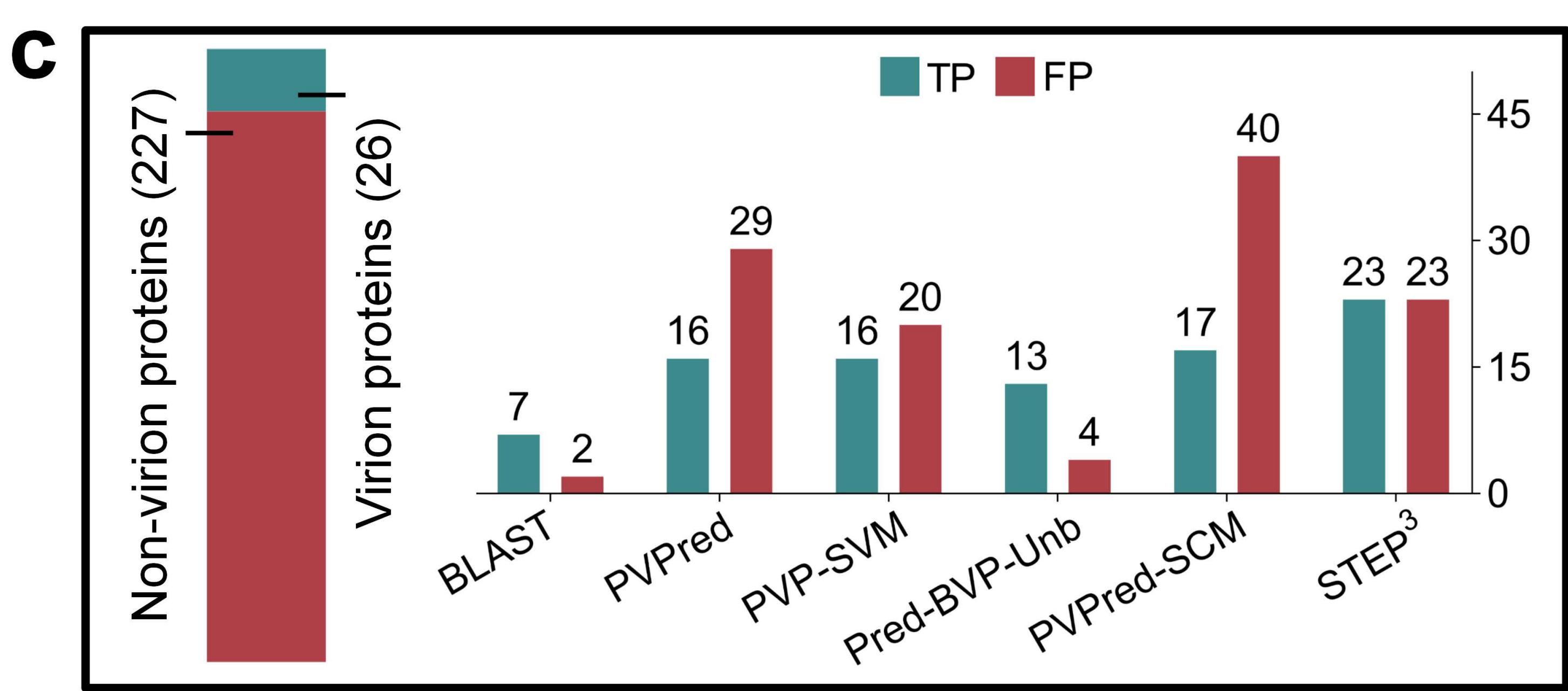

d

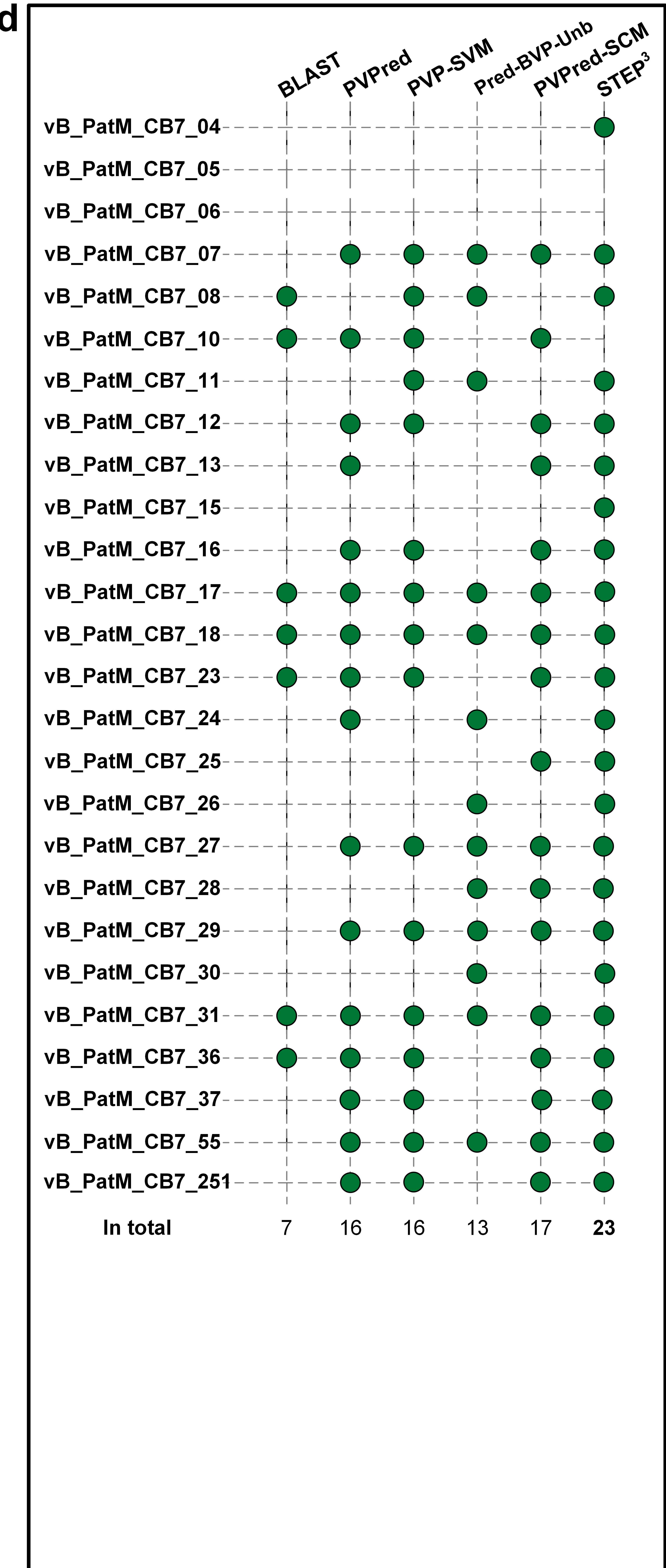

Phage: vB Eco4M-7

Host: Escherichia coli O157
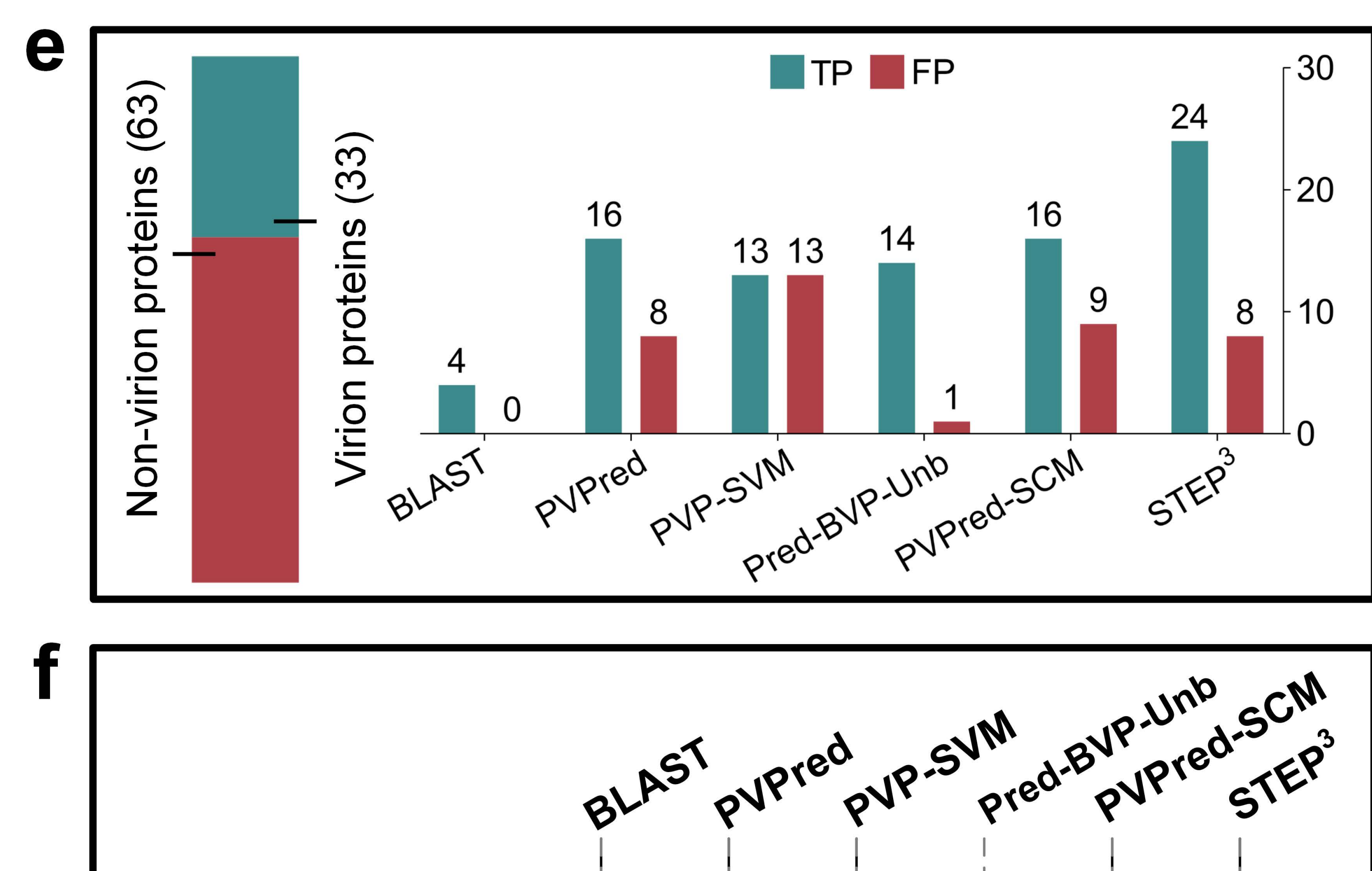

vB_Eco4M-7_09

vB_Eco4M-7_16

VB_Eco4M-7_19

VB_Eco4M-7_26

vB_Eco4M-7_27

vB_Eco4M-7_32

vB_Eco4M-7_36

vB_Eco4M-7_37

vB_Eco4M-7_38

vB_Eco4M-7_40

VB_Ec04M-7_41

vB_Eco4M-7_42

vB_Eco4M-7_45

vB_Ec04M-7_46

vB_Eco4M-7_47

vB_Eco4M-7_48

vB_Eco4M-7_49

vB_Ec04M-7_51

vB_Eco4M-7_52

vB_Ec04M-7_53

vB_Ec04M-7_54

vB_Eco4M-7_57

vB_Eco4M-7_58

vB_Ec04M-7_60

vB_Ec04M-7_61

vB_Ec04M-7_62

vB_Eco4M-7_63

vB_Eco4M-7_64

VB_Eco4M-7_70

VB_Eco4M-7_71

vB_Ec04M-7_73

VB_Eco4M-7_77

vB_Eco4M-7_83

In total

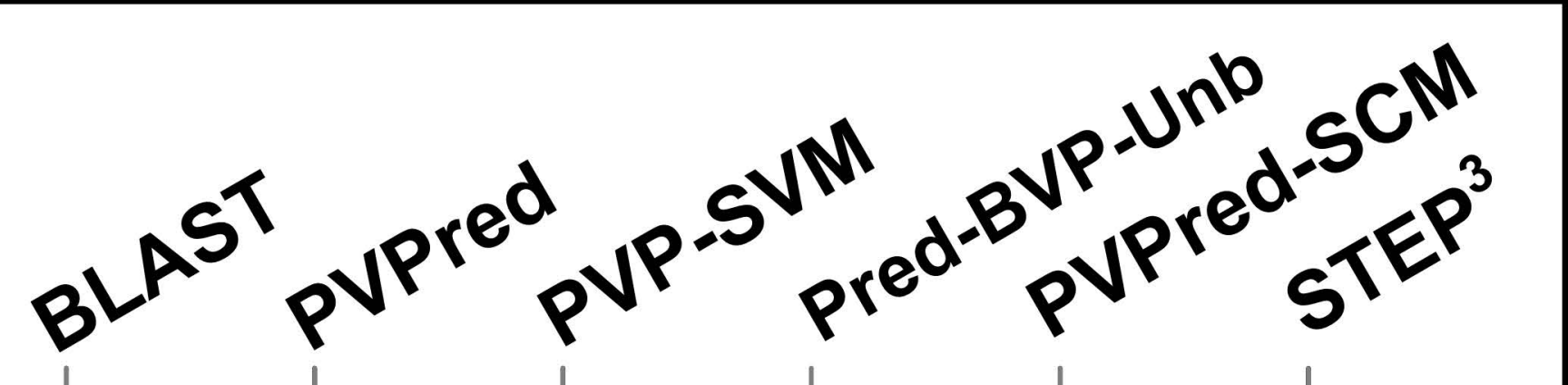

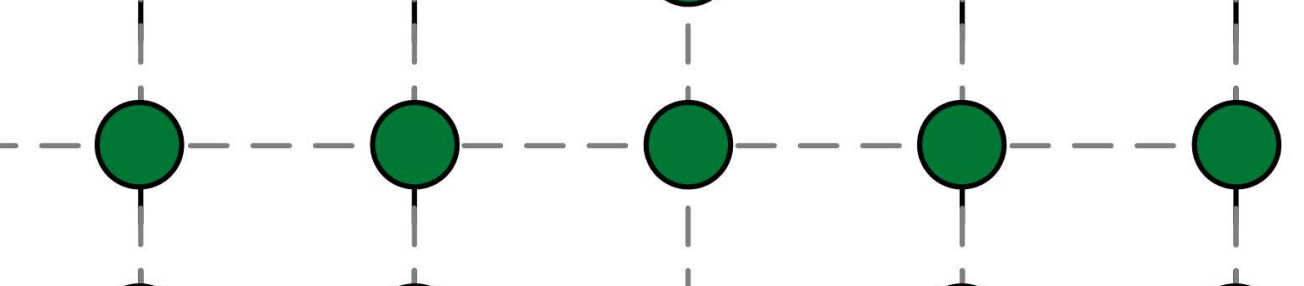

$\varphi^{--} \varphi^{---} \frac{1}{1}---Q^{--} \mathrm{Q}$

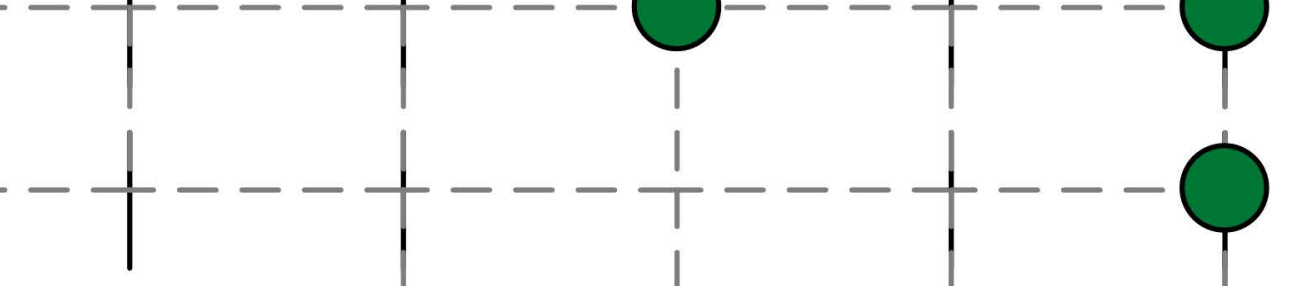
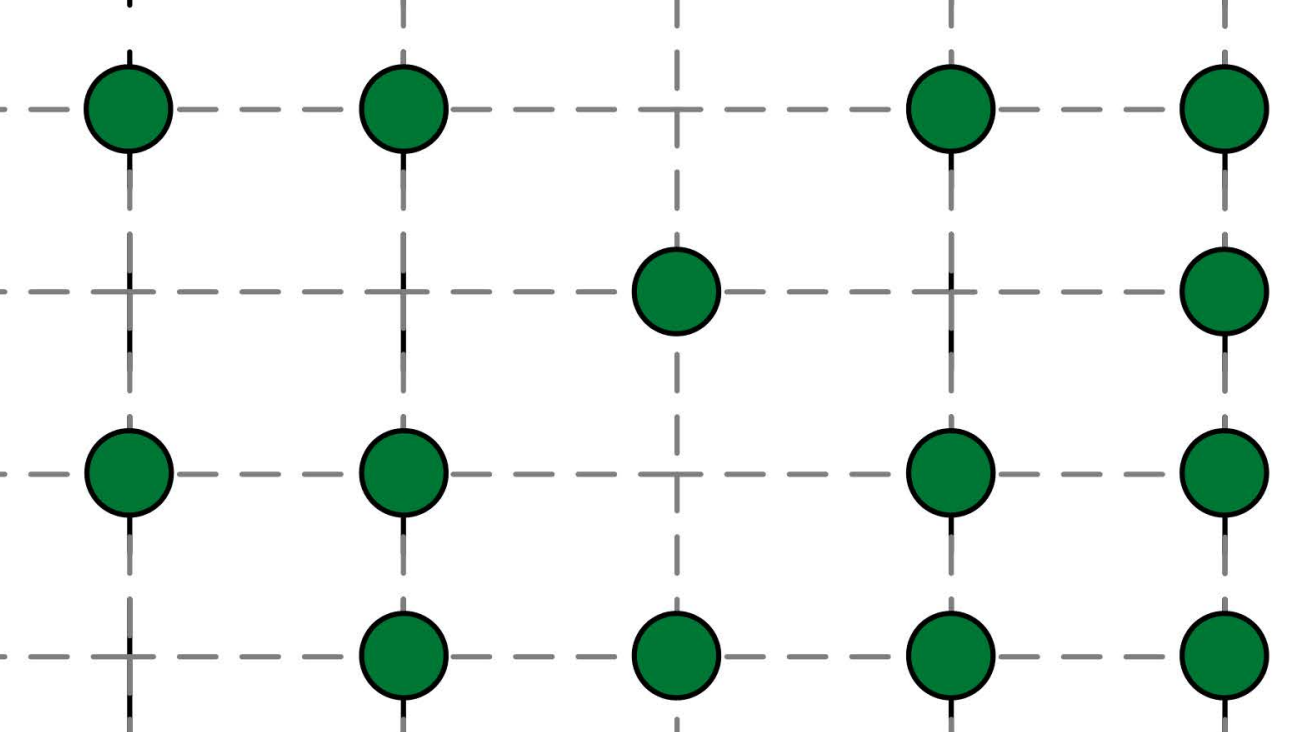

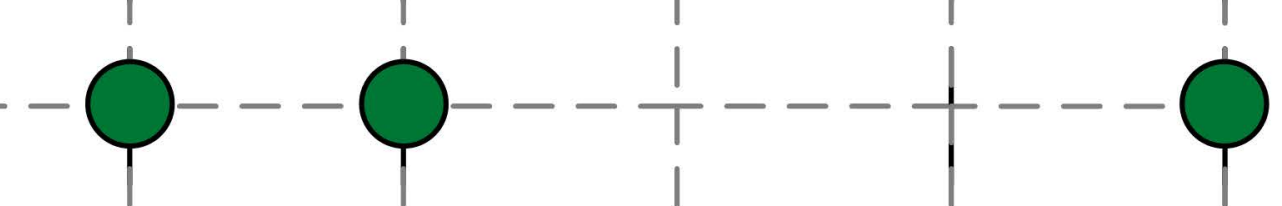

$-0$

$-1-0$

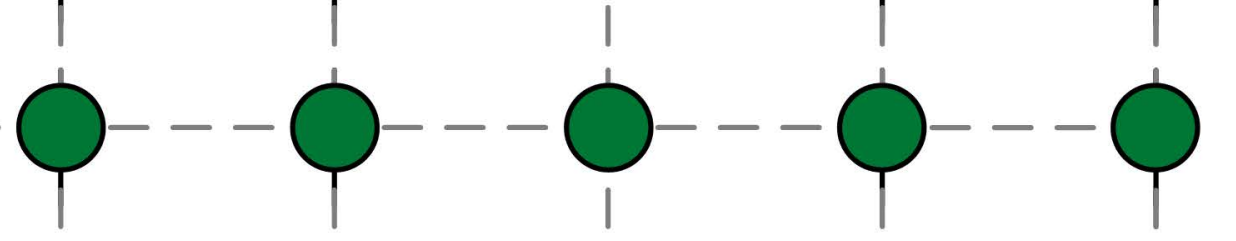

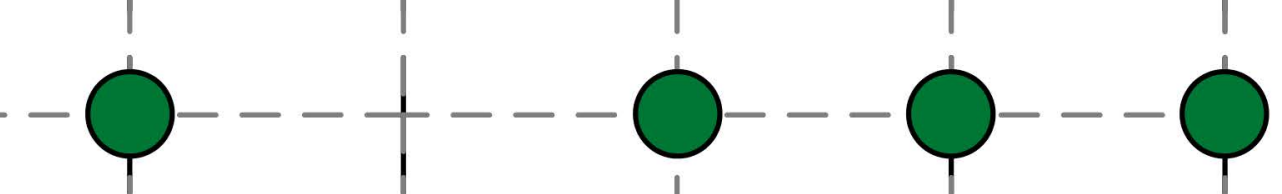

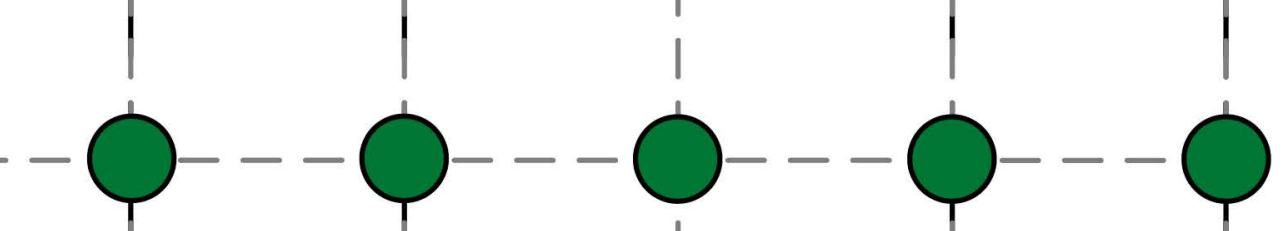

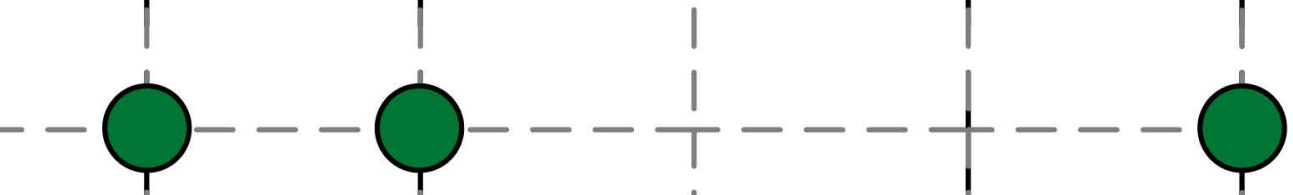

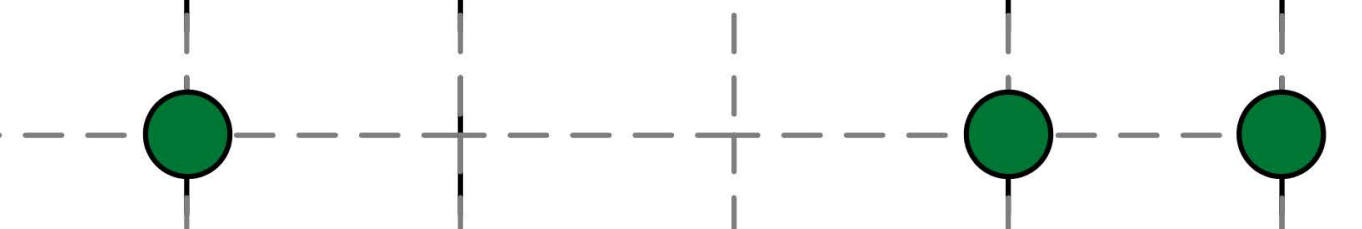

$-\mathrm{O}^{--} \mathrm{O}-\mathrm{O}^{--} \mathrm{O}-\mathrm{O}^{--}$

$十^{---+--O}---\mathrm{O}^{--} \mathrm{O}$

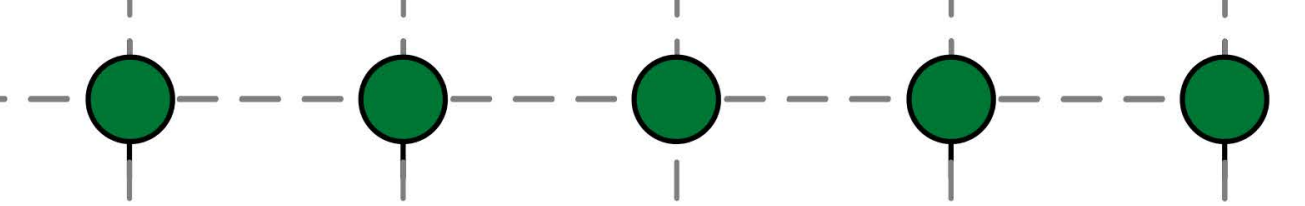

$-\mathrm{O}^{--} \mathrm{O}-\mathrm{O}^{---O}--\mathrm{Q}$

$+-\mathrm{O}_{1}^{---} \mathrm{O}^{---} \mathrm{P}$
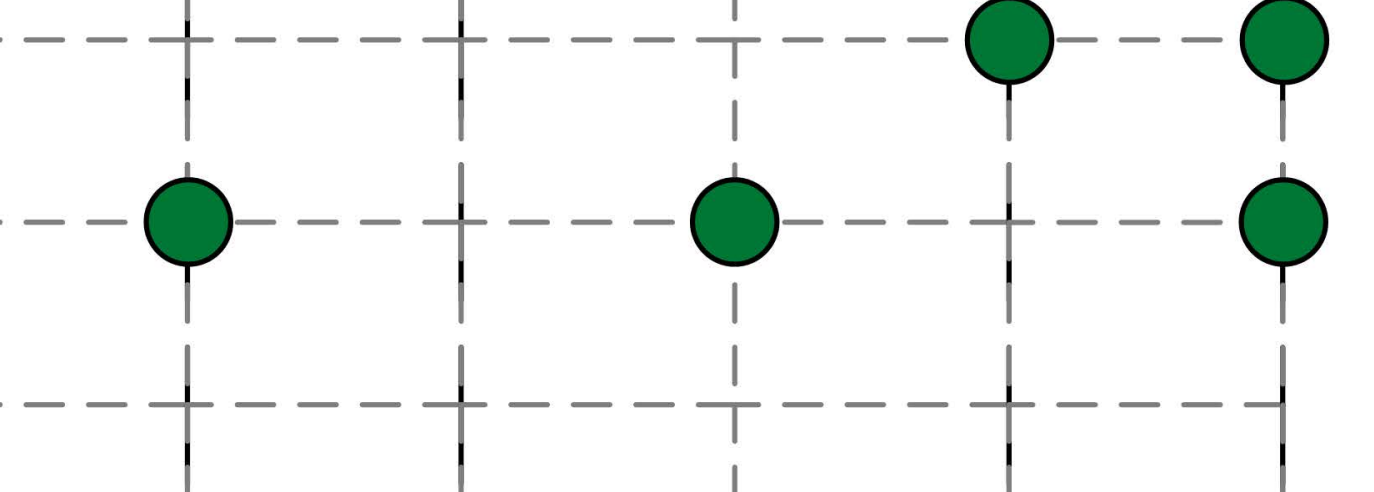

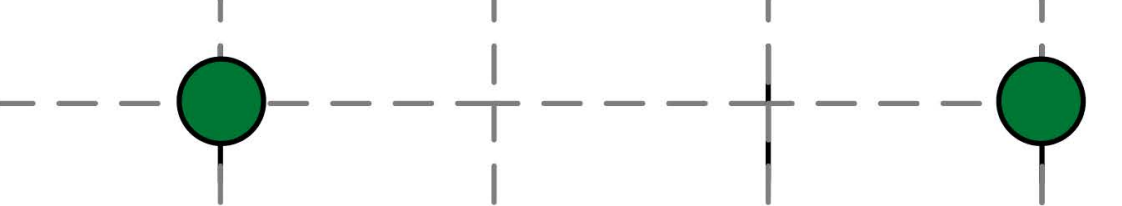

$\left.\right|_{0} ^{30}-20$ 


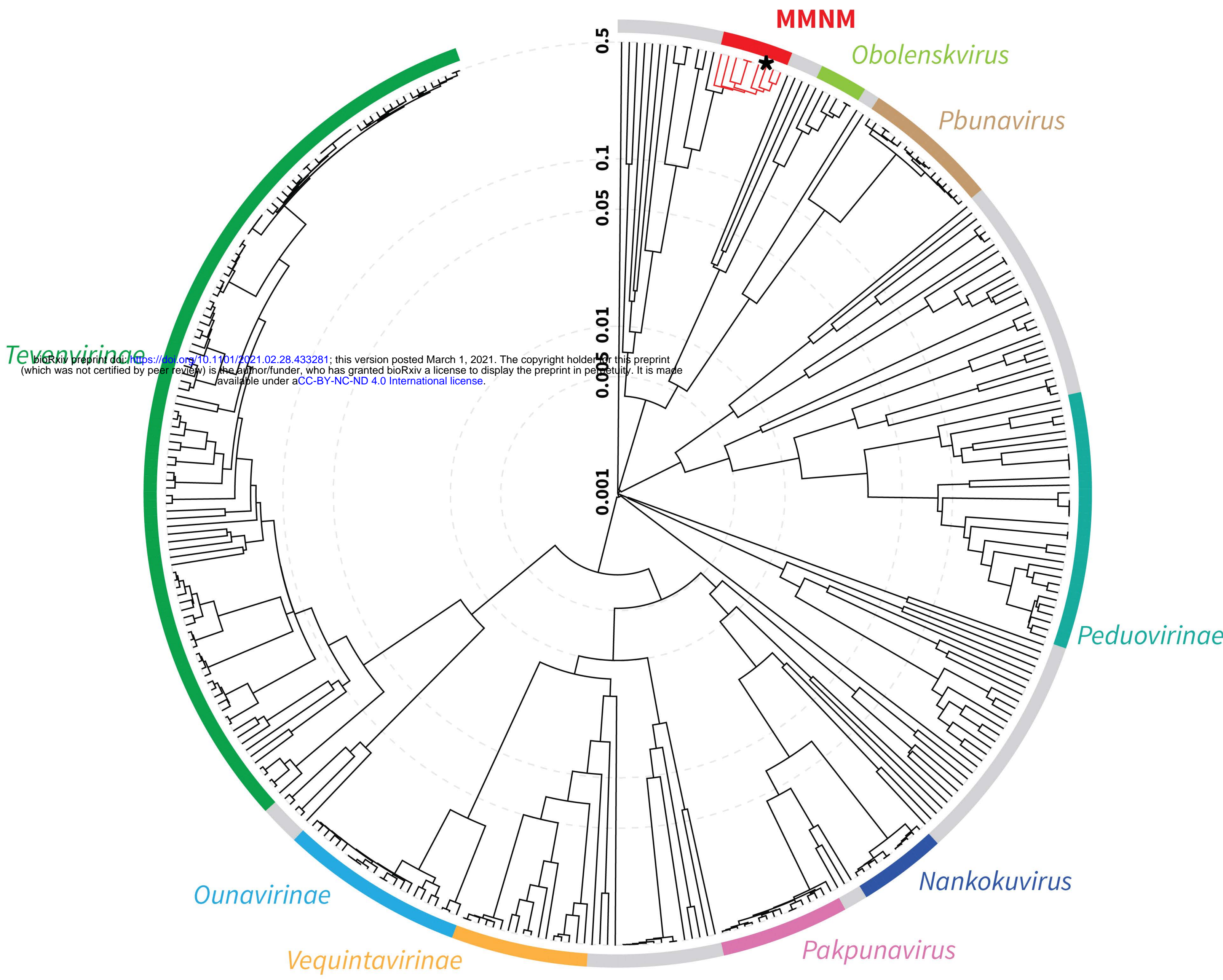

b $47,129 \mathrm{nt}$
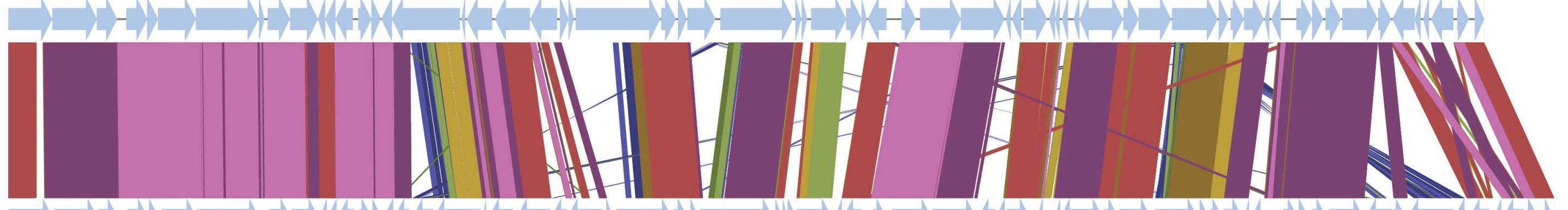

vB_KpnM_FZ14

$49,370 \mathrm{nt}$

vB_KpnM_KpV52

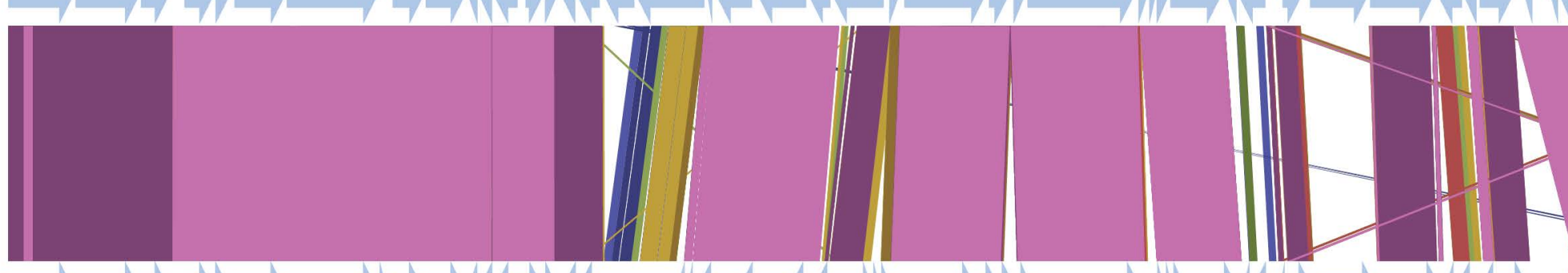

$47,405 \mathrm{nt}$
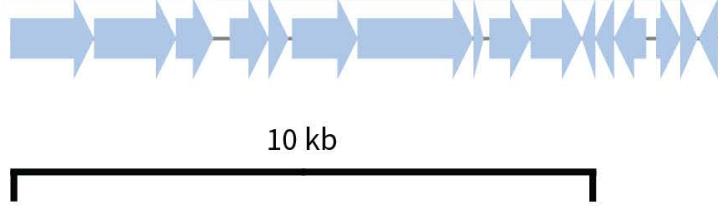

\%-identity

$20 \quad 30$

40
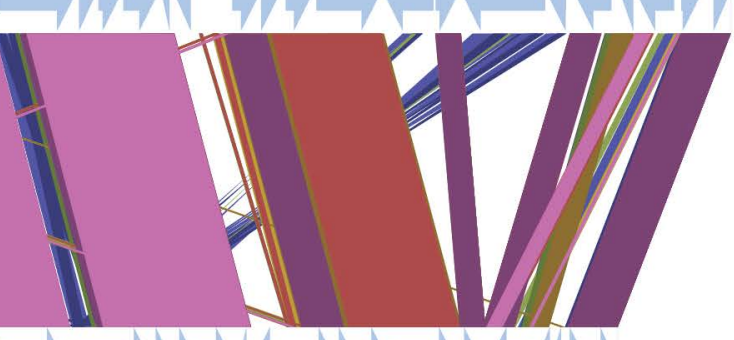

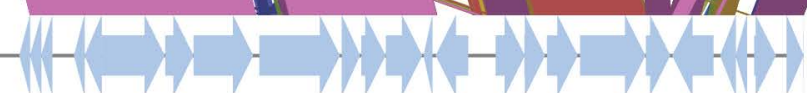


a

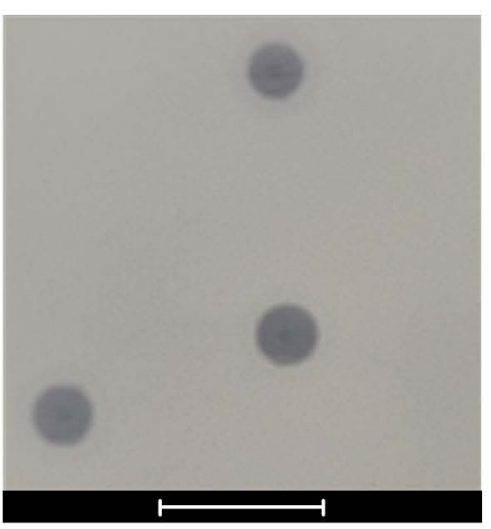

b

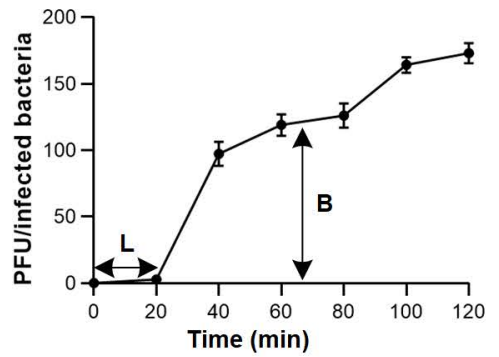

C

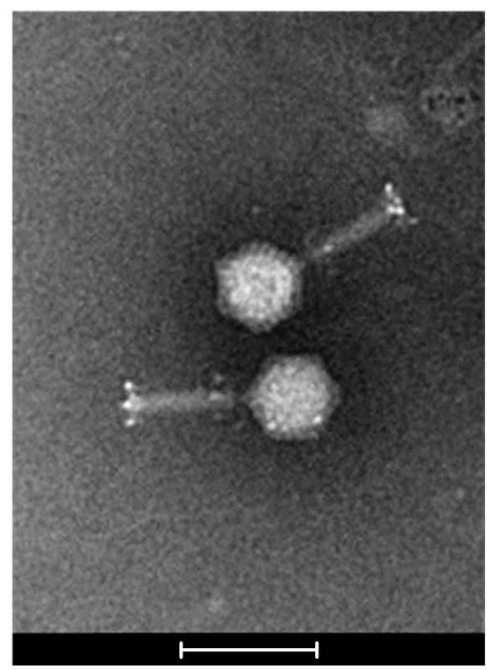

d

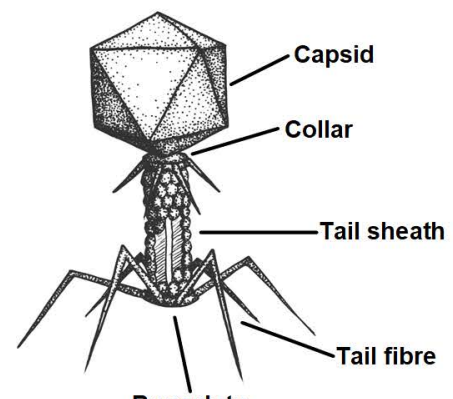

Baseplate
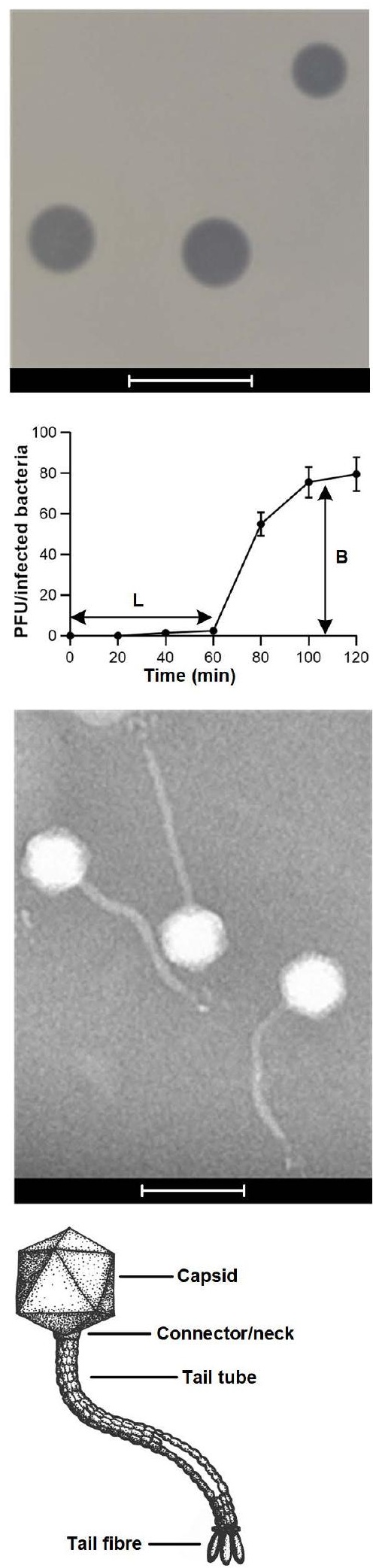
a

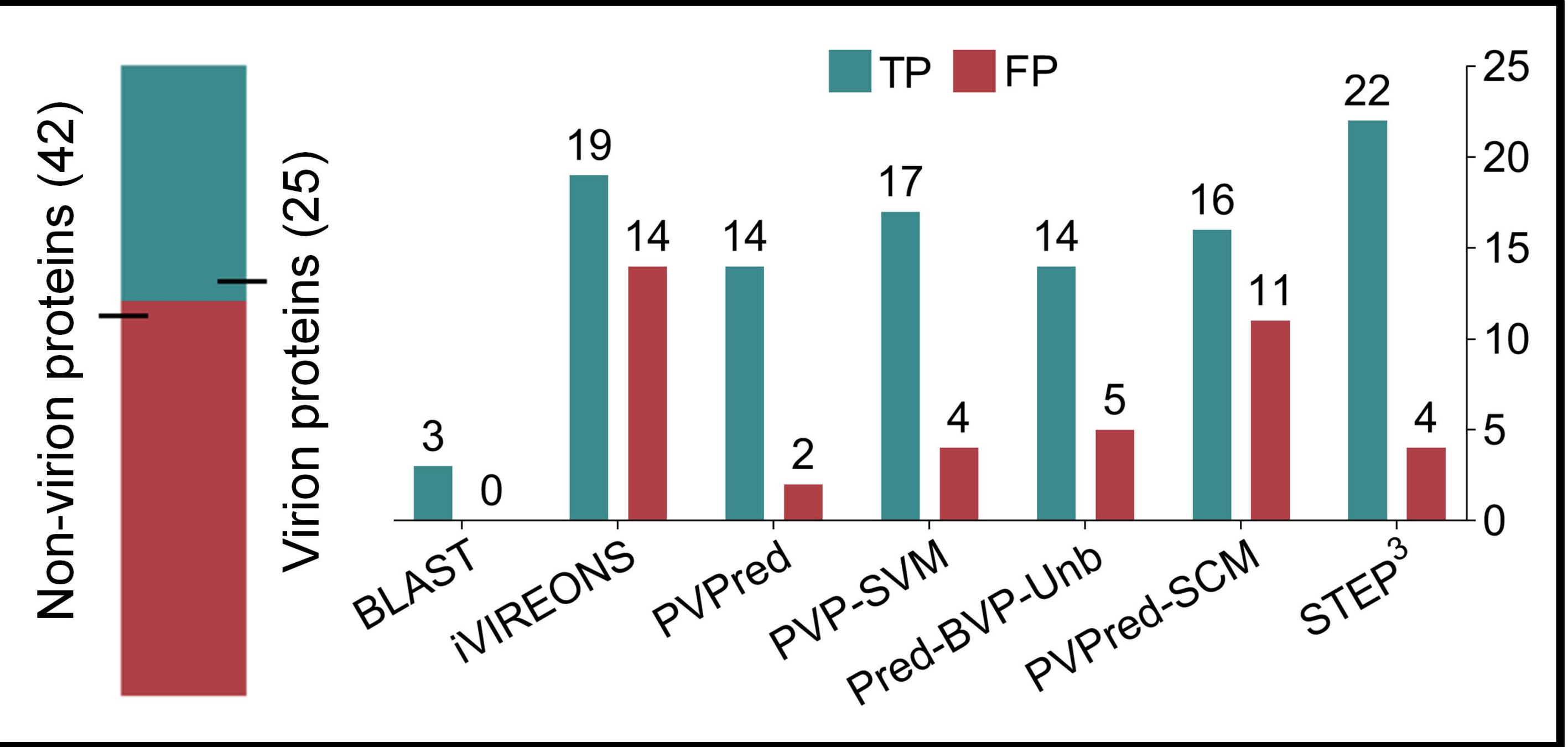

b

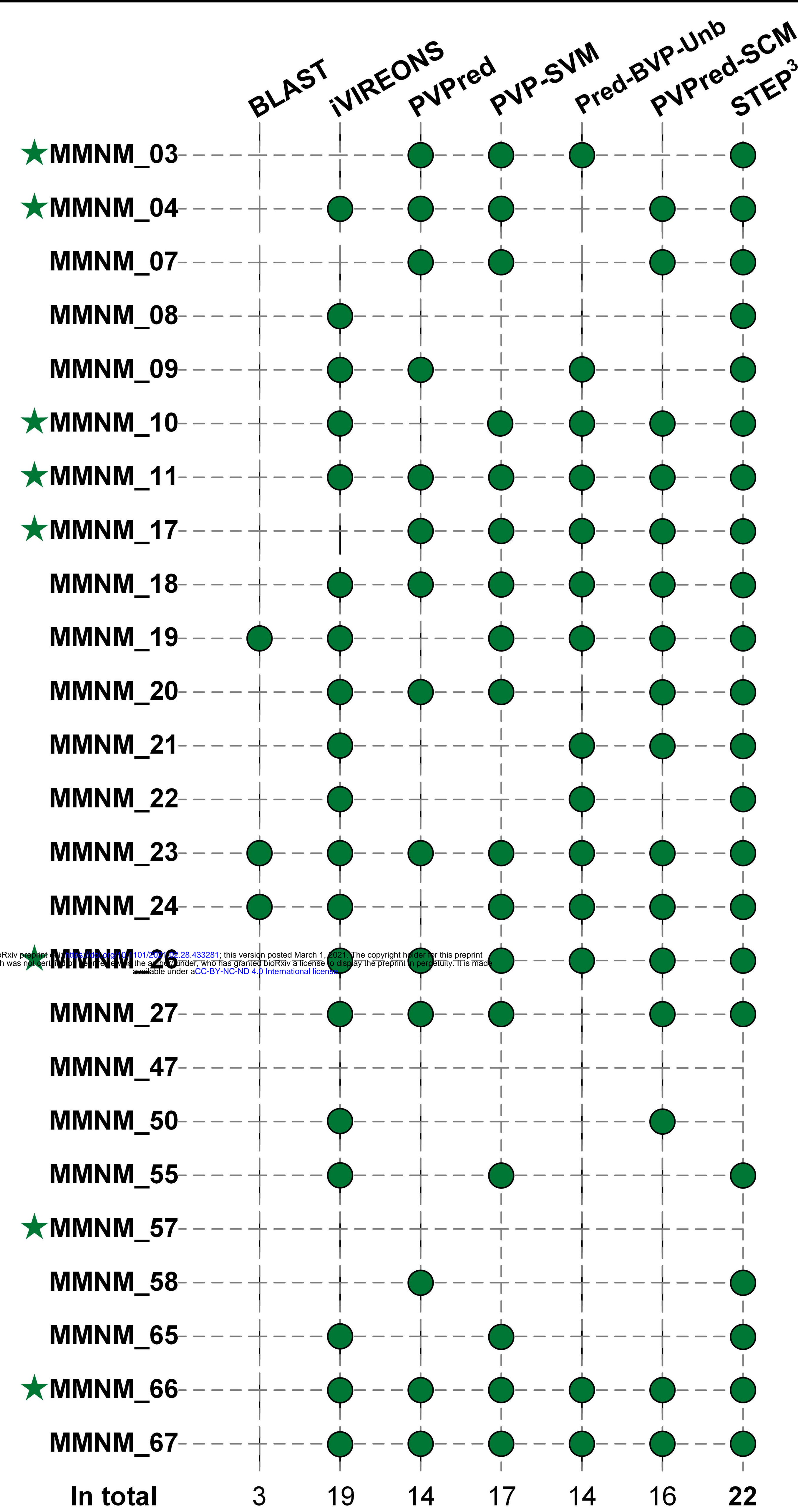

C

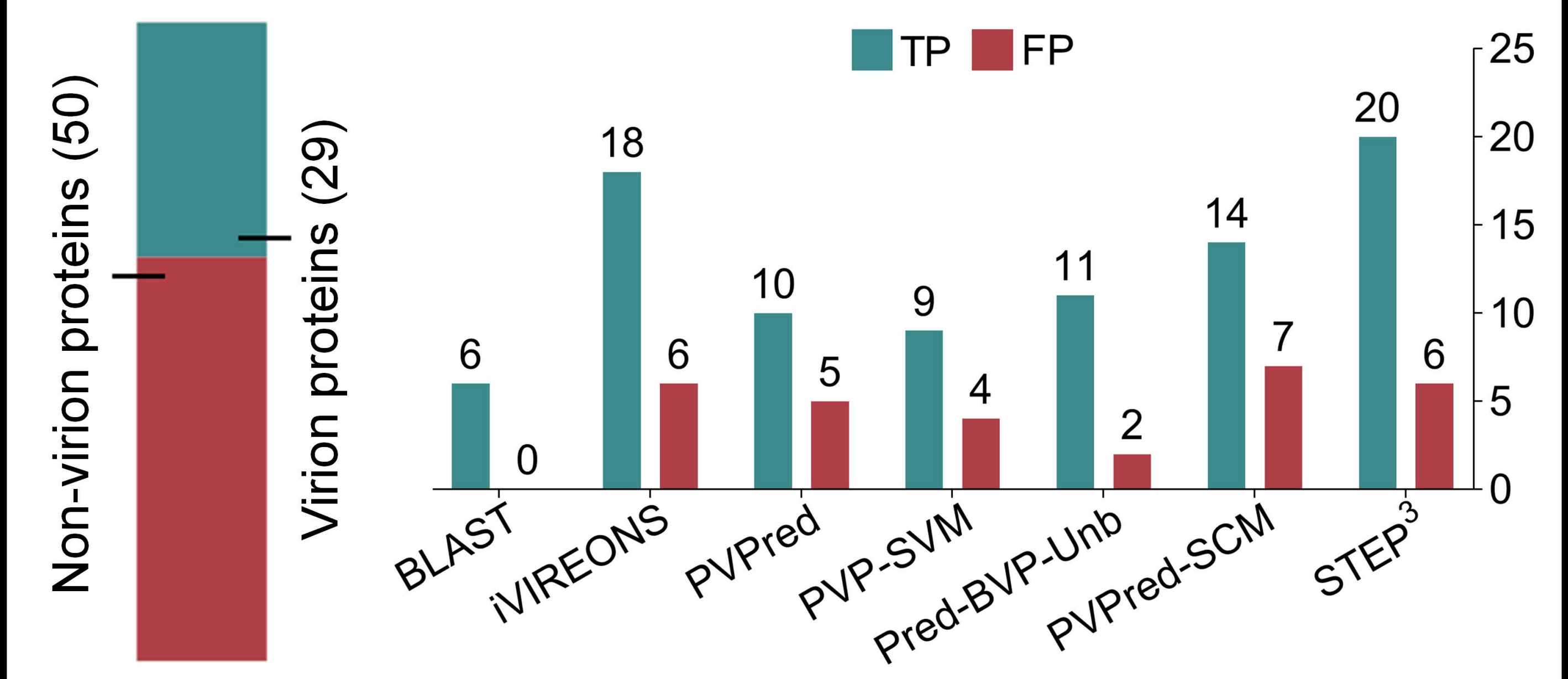

d

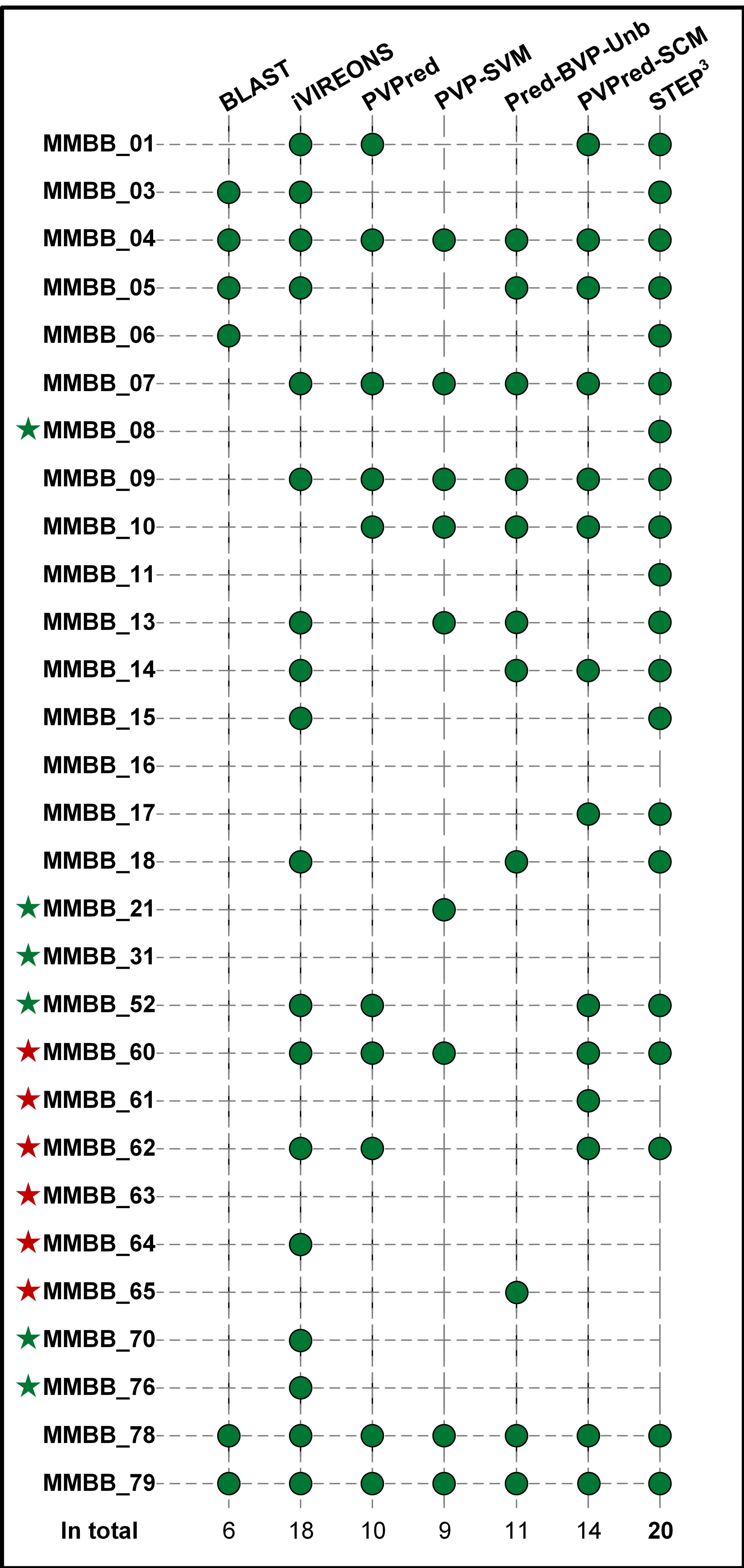

